\title{
AK-TYPE STABILITY THEOREMS ON CROSS $t$-INTERSECTING FAMILIES
}

\author{
SANG JUNE LEE, MARK SIGGERS, AND NORIHIDE TOKUSHIGE
}

\begin{abstract}
Two families, $\mathcal{A}$ and $\mathcal{B}$, of subsets of $[n]$ are cross $t$-intersecting if for every $A \in \mathcal{A}$ and $B \in \mathcal{B}, A$ and $B$ intersect in at least $t$ elements. For a real number $p$ and a family $\mathcal{A}$ the product measure $\mu_{p}(\mathcal{A})$ is defined as the sum of $p^{|A|}(1-p)^{n-|A|}$ over all $A \in \mathcal{A}$. For every non-negative integer $r$, and for large enough $t$, we determine, for any $p$ satisfying $\frac{r}{t+2 r-1} \leq p \leq \frac{r+1}{t+2 r+1}$, the maximum possible value of $\mu_{p}(\mathcal{A}) \mu_{p}(\mathcal{B})$ for cross $t$-intersecting families $\mathcal{A}$ and $\mathcal{B}$. In this paper we prove a stronger stability result which yields the above result.
\end{abstract}

\section{INTRODUCTION}

Let $n \geq t$ be positive integers. Let $[n]:=\{1,2, \ldots, n\}$ and $2^{[n]}:=\{F: F \subset[n]\}$. A family of subsets $\mathcal{A} \subset 2^{[n]}$ is called $t$-intersecting if $\left|A \cap A^{\prime}\right| \geq t$ for all $A, A^{\prime} \in \mathcal{A}$. For any real number $p \in(0,1)$ and a family $\mathcal{A} \subset 2^{[n]}$, we define the product measure $\mu_{p}$ of $\mathcal{A}$ by

$$
\mu_{p}(\mathcal{A}):=\sum_{A \in \mathcal{A}} p^{|A|}(1-p)^{n-|A|} .
$$

What is the maximum product measure of $t$-intersecting families? To answer this question, let $r$ be a non-negative integer and let

$$
\mathcal{F}_{r}^{t}:=\{F \subset[n]:|F \cap[t+2 r]| \geq t+r\} .
$$

The family $\mathcal{F}_{r}^{t}$ is $t$-intersecting since $\left|F \cap F^{\prime} \cap[t+2 r]\right| \geq t$ for all $F, F^{\prime} \in \mathcal{F}_{r}^{t}$. Two families $\mathcal{A}, \mathcal{A}^{\prime} \subset 2^{[n]}$ are isomorphic, denoted by $\mathcal{A} \cong \mathcal{A}^{\prime}$, if $\mathcal{A}^{\prime}=\{\{\pi(a): a \in A\}: A \in$ $\mathcal{A}\}$, where $\pi$ is a permutation on $[n]$. Answering a conjecture of Frankl, and extending partial results by Frankl and Füredi in [12, the following result is essentially proved in [1] by Ahlswede and Khachatrian, see also [2, 6, 9, 16].

Theorem 1. If $\mathcal{A} \subset 2^{[n]}$ is t-intersecting, then

$$
\mu_{p}(\mathcal{A}) \leq \max _{0 \leq r \leq \frac{n-t}{2}} \mu_{p}\left(\mathcal{F}_{r}^{t}\right) .
$$

Moreover, equality holds if and only if $\mathcal{A} \cong \mathcal{F}_{r}^{t}$ for some $r$.

Key words and phrases. Cross intersecting families; Erdős-Ko-Rado theorem; AhlswedeKhachatrian theorem; Shifting; Random walks.

This is a preprint version of the paper appearing in European J. Combinat. 82 (2019) 102993. https://doi.org/10.1016/j.ejc.2019.07.004

The first author was supported by Korea Electric Power Corporation (Grant number:R18XA01) and by Basic Science Research Program through the National Research Foundation of Korea (NRF) funded by the Ministry of Education (NRF-2016R1D1A1B03933404 and NRF-2019R1F1A1058860). The second author is supported by Korean NRF Basic Science Research Program (NRF2018R1D1A1A09083741) and by the Kyungpook National University Research Fund. The last author was supported by JSPS KAKENHI 25287031, 18K03399. 
Grouping the subsets in the family $\mathcal{F}_{r}^{t}$ according to the size of their intersection with $[t+2 r]$ we see, where $q=1-p$,

$$
\mu_{p}\left(\mathcal{F}_{r}^{t}\right)=\sum_{i=0}^{r}\left(\begin{array}{c}
t+2 r \\
i
\end{array}\right) p^{t+2 r-i} q^{i} .
$$

By comparing $\mu_{p}\left(\mathcal{F}_{r+1}^{t} \backslash \mathcal{F}_{r}^{t}\right)=\left(\begin{array}{c}t+2 r \\ r+1\end{array}\right) p^{t+r+1} q^{r+1}$ and $\mu_{p}\left(\mathcal{F}_{r}^{t} \backslash \mathcal{F}_{r+1}^{t}\right)=\left(\begin{array}{c}t+2 r \\ r\end{array}\right) p^{t+r} q^{r+2}$, one sees that $\mu_{p}\left(\mathcal{F}_{r+1}^{t}\right)-\mu_{p}\left(\mathcal{F}_{r}^{t}\right)$ is positive, 0 , negative if and only if $p-\frac{r+1}{t+2 r+1}$ is positive, 0 , negative, respectively. In particular, if

$$
\frac{r}{t+2 r-1} \leq p \leq \frac{r+1}{t+2 r+1}
$$

then

$$
\mu_{p}\left(\mathcal{F}_{0}^{t}\right)<\mu_{p}\left(\mathcal{F}_{1}^{t}\right)<\cdots<\mu_{p}\left(\mathcal{F}_{r-1}^{t}\right) \leq \mu_{p}\left(\mathcal{F}_{r}^{t}\right) \geq \mu_{p}\left(\mathcal{F}_{r+1}^{t}\right)>\mu_{p}\left(\mathcal{F}_{r+2}^{t}\right)>\cdots
$$

Thus the Ahlswede-Khachatrian theorem says that the maximum product measure of $t$-intersecting families is given by $\mu_{p}\left(\mathcal{F}_{r}^{t}\right)$ provided that $t, p$, and $r$ satisfy (2).

We extend this result to two families in $2^{[n]}$. Two families $\mathcal{A}, \mathcal{B} \subset 2^{[n]}$ are cross $t$-intersecting if $|A \cap B| \geq t$ for all $A \in \mathcal{A}$ and $B \in \mathcal{B}$. In this case, it is conjectured in [15] that

$$
\mu_{p}(\mathcal{A}) \mu_{p}(\mathcal{B}) \leq \mu_{p}\left(\mathcal{F}_{r}^{t}\right)^{2}
$$

under the assumption of (2). The inequality (3) was proved for $r=0$ and $n \geq t \geq 14$ in 13 and for $r=1$ and $n \geq t \geq 200$ in [15. We also mention that Borg obtained related results in [3, 4, 5].

In this paper, using the random-walk method that was introduced by Frankl in 10, 11, we verify that this conjecture holds for every fixed $r$ and large $t$. That is we prove the following, referring to [13] for the case $r=0$.

Theorem 2. For every integer $r \geq 0$, there exists an integer $t_{0}=t_{0}(r)$, depending only on $r$, such that for all $n \geq t \geq t_{0}(r)$ and all $p$ with $\frac{r}{t+2 r-1} \leq p \leq \frac{r+1}{t+2 r+1}$, the following holds. If $\mathcal{A}, \mathcal{B} \subset 2^{[n]}$ are cross t-intersecting, then

$$
\mu_{p}(\mathcal{A}) \mu_{p}(\mathcal{B}) \leq \mu_{p}\left(\mathcal{F}_{r}^{t}\right)^{2}
$$

Moreover, equality holds if and only if one of the following holds:

(i) $\mathcal{A}=\mathcal{B} \cong \mathcal{F}_{r-1}^{t}$ and $p=\frac{r}{t+2 r-1}$,

(ii) $\mathcal{A}=\mathcal{B} \cong \mathcal{F}_{r}^{t}$ and $\frac{r}{t+2 r-1} \leq p \leq \frac{r+1}{t+2 r+1}$, or

(iii) $\mathcal{A}=\mathcal{B} \cong \mathcal{F}_{r+1}^{t}$ and $p=\frac{r+1}{t+2 r+1}$.

In this paper we do not attempt to optimize $t_{0}(r)$. We simplify calculations by assuming, for each fixed $r$, that $t$ is sufficiently large. As such, when we use asymptotic notation such as $o(1), O(f)$, or $f \ll g$, it is always asymptotic in $t$, with $r$ fixed, and $p$ being some fixed proportion of the way through the range (2).

We also consider the stability of extremal structures. Suppose that $\mathcal{A}, \mathcal{B} \subset 2^{[n]}$ are cross $t$-intersecting families. If condition (2) is satisfied and $\mu_{p}(\mathcal{A}) \mu_{p}(\mathcal{B})$ is close to the maximum value, then we can ask whether $\mathcal{A}$ and $\mathcal{B}$ are close to (isomorphic copies of) the extremal families $\mathcal{F}_{r-1}^{t}, \mathcal{F}_{r}^{t}$, or $\mathcal{F}_{r+1}^{t}$, where we say that two families $\mathcal{F}$ and $\mathcal{G}$ are close if their symmetric difference $\mathcal{F} \triangle \mathcal{G}=(\mathcal{F} \backslash \mathcal{G}) \cup(\mathcal{G} \backslash \mathcal{F})$ has small measure. We are able to show that this is true if the two families satisfy the additional condition of being 'shifted.' 
A family $\mathcal{A} \subset 2^{[n]}$ is shifted (sometimes called compressed) if $A \in \mathcal{A}$ and $\{i, j\} \cap A=$ $\{j\}$ for some $1 \leq i<j \leq n$ imply $(A \backslash\{j\}) \cup\{i\} \in \mathcal{A}$. It is known (see, e.g., Lemma 2.3 in [13]) that for any given cross $t$-intersecting families $\mathcal{A}, \mathcal{B} \subset 2^{[n]}$ one can apply a sequence of shifting operations to them and get shifted cross $t$-intersecting families $\mathcal{A}^{\prime}, \mathcal{B}^{\prime} \subset 2^{[n]}$ such that $\mu_{p}(\mathcal{A})=\mu_{p}\left(\mathcal{A}^{\prime}\right)$ and $\mu_{p}(\mathcal{B})=\mu_{p}\left(\mathcal{B}^{\prime}\right)$. Notice that the definition of a shifted family depends on the ordering of $[n]$, so an isomorphic copy of a shifted family is not necessarily shifted in this sense.

A family $\mathcal{A}$ is inclusion maximal if $A \subset A^{\prime}$ and $A \in \mathcal{A}$ imply $A^{\prime} \in \mathcal{A}$ as well. Since we are interested in the maximum measure of cross $t$-intersecting families, we always assume that families are inclusion maximal. It is not difficult to see that the property of being inclusion maximal is invariant under shifting operations.

Two families $\mathcal{A}$ and $\mathcal{B}$ are $t$-nice if they are shifted, inclusion maximal, and cross $t$-intersecting. We obtain the following statement of stability.

Theorem 3. For every integer $r \geq 0$ and all real numbers $\epsilon \in(0,1 / 2)$ and $C>2$, there exists an integer $t_{0}=t_{0}(r, \epsilon, C)$ such that for all $n \geq t \geq t_{0}$ the following holds. Let $\frac{r+\epsilon}{t} \leq p \leq \frac{r+1-\epsilon}{t}$, and let $\mathcal{A}, \mathcal{B} \subset 2^{[n]}$ be t-nice families. If

$$
\mu_{p}(\mathcal{A}) \mu_{p}(\mathcal{B}) \geq(1-\delta) \mu_{p}\left(\mathcal{F}_{r}^{t}\right)^{2}
$$

with $\delta \in(0, \epsilon /(r+1))$, then

$$
\mu_{p}\left(\mathcal{A} \triangle \mathcal{F}_{r}^{t}\right)+\mu_{p}\left(\mathcal{B} \triangle \mathcal{F}_{r}^{t}\right) \leq C(1-\sqrt{1-\delta}) \mu_{p}\left(\mathcal{F}_{r}^{t}\right) .
$$

For $r=0$, a similar result was proved in [13]; for the same $t$ it is weaker than this, but it is proved for $t_{0}=14$. There are some points about this theorem that bear further explanation. For one, no matter how close we require that $\mu_{p}(\mathcal{A}) \mu_{p}(\mathcal{B})$ be to $\mu_{p}\left(\mathcal{F}_{r}^{t}\right)^{2}$ in $(5)$ there are $t$-nice families $\mathcal{A}$ and $\mathcal{B}$, which are not subfamilies of $\mathcal{F}_{r}^{t}$, that satisfy (6). Indeed, consider the families

$$
\mathcal{A}=\mathcal{B}=\left(\mathcal{F}_{r}^{t} \backslash\left(\begin{array}{c}
{[t+2 r]} \\
t+r
\end{array}\right)\right) \sqcup\left\{G \sqcup[t+2 r+1, n]: G \in\left(\begin{array}{c}
{[t+2 r]} \\
t+r-1
\end{array}\right)\right\} .
$$

The families $\mathcal{A}$ and $\mathcal{B}$ are $t$-nice, and $\mu_{p}(\mathcal{A}) \mu_{p}(\mathcal{B}) \rightarrow \mu_{p}\left(\mathcal{F}_{r}^{t}\right)^{2}$ for $n \gg t$ as $t \rightarrow \infty$. Theorem 3 says that such families $\mathcal{A}$ and $\mathcal{B}$ must be close to $\mathcal{F}_{r}^{t}$ in the sense that the sum of their measures $\mu_{p}\left(\mathcal{A} \triangle \mathcal{F}_{r}^{t}\right)+\mu_{p}\left(\mathcal{B} \triangle \mathcal{F}_{r}^{t}\right)$ goes to 0 . Observe that with such a definition of closeness, inequality (6) is sharp. Indeed, if $\mathcal{A}=\mathcal{B} \subset \mathcal{F}_{r}^{t}$ and $\mu_{p}(\mathcal{A})=\sqrt{1-\delta} \mu_{p}\left(\mathcal{F}_{r}^{t}\right)$ then the LHS of $(6)$ is precisely $2(1-\sqrt{1-\delta}) \mu_{p}\left(\mathcal{F}_{r}^{t}\right)$.

Another point we should explain is the reduced range of $p$ in the statement of the theorem. When $p=(r+1) /(t+2 r+1)$ we have $\mu_{p}\left(\mathcal{F}_{r+1}^{t}\right)=\mu_{p}\left(\mathcal{F}_{r}^{t}\right)$, so to make a statement of stability with respect to $\mathcal{F}_{r}^{t}$ it is necessary to move $p$ away from this point. We thus introduce a gap of $\epsilon$ into the bound $p<(r+1-\epsilon) /(t+2 r+1)$, and once this is introduced, we absorb constants into it and simplify it to $p<(r+1-\epsilon) / t$. Similarly, we require $p>(r+\epsilon) / t$ because of the family $\mathcal{F}_{r-1}^{t}$. As $\epsilon$ goes to 0 , we must introduce $\delta$ in (5) that depends on $\epsilon$.

It turns out that the condition $\delta<\epsilon /(r+1)$ is sharp. Indeed, consider the following pair of families:

$$
\mathcal{A}=\mathcal{F}_{r+1}^{t-1} \text { and } \mathcal{B}=\mathcal{F}_{r}^{t+1} \text {. }
$$

The families $\mathcal{A}$ and $\mathcal{B}$ are $t$-nice, and far from $\mathcal{F}_{r}^{t}$, in fact, both $\mu_{p}\left(\mathcal{A} \backslash \mathcal{F}_{r}^{t}\right) / \mu_{p}\left(\mathcal{F}_{r}^{t}\right)$ and $\mu_{p}\left(\mathcal{F}_{r}^{t} \backslash \mathcal{B}\right) / \mu_{p}\left(\mathcal{F}_{r}^{t}\right)$ go to infinity as $t \rightarrow \infty$. On the other hand, one can show 
that if $p=\frac{r+1-\epsilon}{t}$, then

$$
\frac{\mu_{p}(\mathcal{A}) \mu_{p}(\mathcal{B})}{\mu_{p}\left(\mathcal{F}_{r}^{t}\right)^{2}}=\left(1-\frac{\epsilon}{r+1}\right)(1+o(1))
$$

(See 12$)$ ). So the condition $\delta<\epsilon /(r+1)$ cannot be improved.

For a full stability result, we must consider these other extremal families. We do this with the more complicated Theorem 4 , from which Theorem 3 follows as a corollary. Assume that $\mathcal{A}$ and $\mathcal{B}$ are $t$-nice families, and $(2)$ is satisfied. Theorem 4 says that either $\mu_{p}(\mathcal{A}) \mu_{p}(\mathcal{B})$ is much smaller than the optimal value $\mu_{p}\left(\mathcal{F}_{r}^{t}\right)^{2}$, or $\{\mathcal{A}, \mathcal{B}\}$ is close to one of

$$
\left\{\mathcal{F}_{r-1}^{t}, \mathcal{F}_{r-1}^{t}\right\},\left\{\mathcal{F}_{r}^{t}, \mathcal{F}_{r}^{t}\right\},\left\{\mathcal{F}_{r+1}^{t}, \mathcal{F}_{r+1}^{t}\right\},\left\{\mathcal{F}_{r}^{t-1}, \mathcal{F}_{r-1}^{t+1}\right\}, \text { or }\left\{\mathcal{F}_{r+1}^{t-1}, \mathcal{F}_{r}^{t+1}\right\} .
$$

We denote the set of pairs of subscripts of the extremal families in (7) by

$$
R_{\mathrm{ex}}:=\left\{\begin{array}{cc}
\{(0,0),(1,1),(1,0)\} & \text { if } r=0 \\
\{(r-1, r-1),(r, r),(r+1, r+1),(r, r-1),(r+1, r)\} & \text { if } r \geq 1 .
\end{array}\right.
$$

If $\mu_{p}(\mathcal{A}) \mu_{p}(\mathcal{B})$ is close to optimal, then we will have, up to switching $\mathcal{A}$ and $\mathcal{B}$, that $(\mathcal{A}, \mathcal{B})$ is close to $\left(\mathcal{F}_{s}^{u}, \mathcal{F}_{s^{\prime}}^{v}\right)$ for a unique $\left(s, s^{\prime}\right) \in R_{\mathrm{ex}}$, with $u=t-\left(s-s^{\prime}\right)$ and $v=t+\left(s-s^{\prime}\right)$. Again, to quantify 'close to' we consider the measure of the symmetric differences $\mathcal{A} \triangle \mathcal{F}_{s}^{u}$ and $\mathcal{B} \triangle \mathcal{F}_{s^{\prime}}^{v}$. We could just sum these, but we will observe below, in 200 , that $\mu_{p}\left(\mathcal{F}_{r}^{t}\right)=\Theta\left(p^{t}\right)$, so the measures of $\mathcal{F}_{s}^{u}$ and $\mathcal{F}_{s^{\prime}}^{v}$ can be vastly different. It is natural, therefore, to normalise the measures of these symmetric differences with respect to the measures of $\mathcal{F}_{s}^{u}$ and $\mathcal{F}_{s^{\prime}}^{v}$. We thus define the following normalised measures:

$$
\begin{aligned}
X & :=p^{t-u} \mu_{p}(\mathcal{A})+p^{t-v} \mu_{p}(\mathcal{B}), \\
X_{\mathcal{F}} & :=p^{t-u} \mu_{p}\left(\mathcal{F}_{s}^{u}\right)+p^{t-v} \mu_{p}\left(\mathcal{F}_{s^{\prime}}^{v}\right), \\
X_{\Delta} & :=p^{t-u} \mu_{p}\left(\mathcal{A} \triangle \mathcal{F}_{s}^{u}\right)+p^{t-v} \mu_{p}\left(\mathcal{B} \triangle \mathcal{F}_{s^{\prime}}^{v}\right), \\
X_{*} & :=p^{t-u} \mu_{p}\left(\mathcal{A} \backslash \mathcal{F}_{s}^{u}\right)+p^{t-v} \mu_{p}\left(\mathcal{B} \backslash \mathcal{F}_{s^{\prime}}^{v}\right) .
\end{aligned}
$$

Now we can state our main result.

Theorem 4. For every integer $r \geq 0$, and all real numbers $\epsilon, \epsilon_{1} \in(0,1 / 2)$, and $\delta_{1} \in(0,1 /(r+2))$, there exists an integer

$$
t_{0}=\left\{\begin{array}{l}
t_{0}\left(r, \epsilon_{1}, \delta_{1}\right) \text { for } r \geq 1, \\
t_{0}\left(\epsilon, \epsilon_{1}, \delta_{1}\right) \text { for } r=0
\end{array}\right.
$$

such that for all $n \geq t \geq t_{0}$ the following holds. Let

$$
\frac{r}{t+2 r-1} \leq p \leq \frac{r+1}{t+2 r+1} \text { for } r \geq 1 \text {, and } \frac{\epsilon}{t} \leq p \leq \frac{1}{t+1} \text { for } r=0,
$$

and let $\mathcal{A}, \mathcal{B} \subset 2^{[n]}$ be $t$-nice families. Suppose

$$
\mu_{p}(\mathcal{A}) \mu_{p}(\mathcal{B}) \geq\left(1-\delta_{1}\right) \mu_{p}\left(\mathcal{F}_{r}^{t}\right)^{2} .
$$

Then, up to switching $\mathcal{A}$ and $\mathcal{B}$, there exists unique $\left(s, s^{\prime}\right) \in R_{\mathrm{ex}}$ such that, for

$$
u=t-\left(s-s^{\prime}\right) \text { and } v=t+\left(s-s^{\prime}\right),
$$

the following hold.

(a) $X \leq X_{\mathcal{F}}$ with equality holding if and only if $\mathcal{A}=\mathcal{F}_{s}^{u}$ and $\mathcal{B}=\mathcal{F}_{s^{\prime}}^{v}$,

(b) $X_{*} \leq \epsilon_{1} X_{\Delta}$, and 
(c) $\mu_{p}(\mathcal{A}) \mu_{p}(\mathcal{B}) \leq \mu_{p}\left(\mathcal{F}_{s}^{u}\right) \mu_{p}\left(\mathcal{F}_{s^{\prime}}^{v}\right)$ with equality holding if and only if $\mathcal{A}=\mathcal{F}_{s}^{u}$ and $\mathcal{B}=\mathcal{F}_{s^{\prime}}^{v}$.

What does this say exactly? It says that if the product of the measures of $\mathcal{A}$ and $\mathcal{B}$ is close to optimal, then $(\mathcal{A}, \mathcal{B})$ has measure not greater than one of the one of the pairs $\left(\mathcal{F}_{s}^{u}, \mathcal{F}_{s^{\prime}}^{v}\right)$ of extremal families in $(7)$, and is close to this pair, in the sense that $X_{\Delta}$ is small. To see that $X_{\Delta}$ is small one can use (b) to show $X_{\Delta} \leq \frac{2\left(1-\sqrt{1-\delta_{1}}\right)}{1-2 \epsilon_{1}} \mu_{p}\left(\mathcal{F}_{r}^{t}\right)$ as we do for 15$)$. That the measure of $(\mathcal{A}, \mathcal{B})$ is at most that of $\left(\mathcal{F}_{s}^{u}, \mathcal{F}_{s^{\prime}}^{v}\right)$ is stated with (a) and (c). We explain why there are two statements. The statements are very similar when (9) holds. Statement (c) is perhaps the more obvious statement, in light of the required inequality (4) of Theorem 2, and in the case that $s=s^{\prime}=r$ it is all we need for proving both Theorem 2 and 3 . Moreover, in this case (c) follows from (a) by the AM-GM inequality. When $s-s^{\prime}=1$ things are not so clean, and we need both statements. Statement (a) is stronger as $p$ approaches $r /(t+2 r-1)$ or $(r+1) /(t+2 r+1)$ and so we use this to prove Theorem 2. When $p$ is bounded away from these endpoints, (c) is stronger (and harder to prove) than statement (a). We use it to prove Theorem 3 .

We mention that the inequality $X \leq X_{\mathcal{F}}$ in (a) is not necessarily true unless $(9)$ holds. Indeed if, e.g., $\{\mathcal{A}, \mathcal{B}\}=\left\{\emptyset, 2^{[n]}\right\}$, or $\left\{\{[t]\}, \bigcup_{i>t}\left(\begin{array}{c}{[n]} \\ t\end{array}\right)\right\}$, then it follows that $X \gg X_{\mathcal{F}}$ if $n \gg t$.

We also mention that the condition $\delta_{1}<\frac{1}{r+2}$ is tight. To see this, let $\{\mathcal{A}, \mathcal{B}\}=$ $\left\{\mathcal{F}_{r+2}^{t-1}, \mathcal{F}_{r+1}^{t+1}\right\}$, or $\left\{\mathcal{F}_{r+2}^{t-2}, \mathcal{F}_{r}^{t+2}\right\}$, and let $p=\frac{r+1}{t+2 r+1}$. Then $\mathcal{A}$ and $\mathcal{B}$ are $t$-nice, and we have $\mu_{p}(\mathcal{A}) \mu_{p}(\mathcal{B}) / \mu_{p}\left(\mathcal{F}_{r}^{t}\right)^{2}>1-\frac{1}{r+2}$, (and the LHS converges to the RHS as $t \rightarrow \infty$, ) but (b) does not hold for any $\left(s, s^{\prime}\right) \in R_{\mathrm{ex}}$. This means that one cannot replace the condition $(9)$ with $\mu_{p}(\mathcal{A}) \mu_{p}(\mathcal{B})>\left(1-\frac{1}{r+2}\right) \mu_{p}\left(\mathcal{F}_{r}^{t}\right)^{2}$.

The organization of this paper is as follows. In Section 2 we derive Theorems 2 and 3 from Theorem 4. Most of the rest of the paper is dedicated to the proof of Theorem 4. In Section 3 we recall some useful tools from [13] and [15. In Section 4.1] we lay out our asymptotic assumptions and use them to give simplified expressions for frequently used values. In Section 4.2 we reduce Theorem 4 to the essential case: $u+v=2 t$. We then define the parameters $s$ and $s^{\prime}$, and use them to distinguish three cases for the proof of Theorem 4 . the non-extremal case, the diagonal extremal case, and the non-diagonal extremal case. In Section 5 we settle the non-extremal case. We deal with the diagonal extremal case in Section 6, and then, following the proof of this very closely, we consider the non-diagonal extremal case in Section 7. In Section 8 we make some brief comments about a recent result of Ellis, Keller and Lifshitz [8] which is related to Theorem 3

\section{Proofs of Theorems 2 and 3}

In this section we derive Theorems 2 and 3 from Theorem 4 . We use some basic asymptotics from Section 4.1.

2.1. Proof of Theorem 2 (using Theorem 4). The case $r=0$ of Theorem 2 is proved in [13, and hence, we fix $r \geq 1$. Let $\epsilon_{1}$ be a fixed constant in $(0,1 / 2)$, and let $t_{0}$ be $t_{0}\left(r, \epsilon_{1}, 1 /(r+3)\right)$ of Theorem 4 . Let $n, t, p$ be chosen so that $n \geq t \geq t_{0}$ and $\frac{r}{t+2 r-1} \leq p \leq \frac{r+1}{t+2 r+1}$. 
We first suppose that $\mathcal{A}, \mathcal{B} \subset 2^{[n]}$ are $t$-nice families. If condition (9) of Theorem 4 does not hold, then Theorem 2 is clearly true, so assume it holds and apply Theorem 4 This gives us values $\left(s, s^{\prime}\right) \in R_{\mathrm{ex}}$.

In the case that $s=s^{\prime}$, we have $u=v=t$. Item (c) of Theorem 4 gives

$$
\mu_{p}(\mathcal{A}) \mu_{p}(\mathcal{B}) \leq \mu_{p}\left(\mathcal{F}_{s}^{u}\right) \mu_{p}\left(\mathcal{F}_{s^{\prime}}^{v}\right)=\mu_{p}\left(\mathcal{F}_{r}^{t}\right)^{2},
$$

where the equality holds if and only if $\mathcal{A}$ and $\mathcal{B}$ satisfy one of (i), (ii), and (iii) of Theorem 2,

In case that $s-s^{\prime}=1$, the AM-GM inequality and (a) of Theorem 4 imply that

$$
2 \sqrt{\mu_{p}(\mathcal{A}) \mu_{p}(\mathcal{B})} \leq X \leq X_{\mathcal{F}}
$$

The following claim immediately implies (4).

Claim 1. For $t \geq t_{0}$ and $\left(s, s^{\prime}\right) \in\{(r, r-1),(r+1, r)\}$ we have

$$
X_{\mathcal{F}}=p \mu_{p}\left(\mathcal{F}_{s}^{u}\right)+p^{-1} \mu_{p}\left(\mathcal{F}_{s^{\prime}}^{v}\right)<2 \mu_{p}\left(\mathcal{F}_{r}^{t}\right) .
$$

Except for proving this claim, we have thus proved Theorem 2 provided that $\mathcal{A}$ and $\mathcal{B}$ are $t$-nice families. Now suppose that $\mathcal{A}$ and $\mathcal{B}$ are (not necessarily shifted) inclusion maximal and cross $t$-intersecting families. Let $\mathcal{A}^{\prime}$ and $\mathcal{B}^{\prime}$ be $t$-nice families obtained from $\mathcal{A}$ and $\mathcal{B}$ after applying a sequence of shifting operations. Then, by the fact we have just proved, the families $\mathcal{A}^{\prime}$ and $\mathcal{B}^{\prime}$ satisfy the inequality (4) with the equality conditions. Since the measure is invariant under shifting operations, we have $\mu_{p}(\mathcal{A}) \mu_{p}(\mathcal{B})=\mu_{p}\left(\mathcal{A}^{\prime}\right) \mu_{p}\left(\mathcal{B}^{\prime}\right)$, and hence, we still have (4) for $\mathcal{A}$ and $\mathcal{B}$. Moreover, it is known from Lemma 6 in [15] that if $\mathcal{A}^{\prime}=\mathcal{B}^{\prime} \cong \mathcal{F}_{y}^{x}$ then $\mathcal{A}=\mathcal{B} \cong \mathcal{F}_{y}^{x}$. Thus the equality conditions hold for $\mathcal{A}$ and $\mathcal{B}$ as well. This completes the proof of Theorem 2 up to the proof of Claim 11, which we give now.

Proof of Claim 1. Since $s=s^{\prime}+1$, we have that $u=t-1$ and $v=t+1$.

First, we consider the case where $\left(s, s^{\prime}\right)=(r, r-1)$. Using $p=\Theta(1 / t)$, it follows from (1) (see also (19p) that

$$
\mu_{p}\left(\mathcal{F}_{r}^{t}\right)=\left(\begin{array}{c}
t+2 r \\
r
\end{array}\right) p^{t+2 r-r} q^{r}\left(1+\Theta\left(1 / t^{2}\right)\right)
$$

Hence, we can check Claim 1 by comparing the main terms of $p \mu_{p}\left(\mathcal{F}_{s}^{u}\right)+p^{-1} \mu_{p}\left(\mathcal{F}_{s^{\prime}}^{v}\right)$ and $2 \mu_{p}\left(\mathcal{F}_{r}^{t}\right)$. That is, it suffices to show $p\left(\begin{array}{c}t+2 r-1 \\ r\end{array}\right) p^{t+r-1} q^{r}+\frac{1}{p}\left(\begin{array}{c}t+2 r-1 \\ r-1\end{array}\right) p^{t+r} q^{r-1}<$ $2\left(\begin{array}{c}t+2 r \\ r\end{array}\right) p^{t+r} q^{r}$, that is, $\left(\begin{array}{c}t+2 r-1 \\ r\end{array}\right)+\left(\begin{array}{c}t+2 r-1 \\ r-1\end{array}\right) \frac{1}{p q}<2\left(\begin{array}{c}t+2 r \\ r\end{array}\right)$, or equivalently, $p(1-p)>$ $\frac{r}{t+3 r}$. The LHS is minimized, for $p$ satisfying (2), at $p=\frac{r}{t+2 r-1}$; and in this case the above inequality is equivalent to $t>r^{2}-r+1$.

Next, we consider the case where $\left(s, s^{\prime}\right)=(r+1, r)$. In this case it suffices to show that $\left(\begin{array}{c}t+2 r+1 \\ r+1\end{array}\right) p q+\left(\begin{array}{c}t+2 r+1 \\ r\end{array}\right)<2\left(\begin{array}{c}t+2 r \\ r\end{array}\right)$, or equivalently, $p(1-p)<\frac{(r+1)(t+1)}{(t+2 r+1)(t+r+1)}$. The LHS is maximized at $p=\frac{r+1}{t+2 r+1}$ and then the above inequality is equivalent to $t>r^{2}-r-1$.

Although we use [13] for the case $r=0$, we could have proved this case with Theorem 4 as well; but it would have required a slightly more complicated statement. The only problem in applying Theorem 4 as is, is the condition $\epsilon / t \leq p$ in the case $r=0$. This condition is used only in proving Lemma 18, which gives us item (c) of Theorem 4 in the case that $s \neq s^{\prime}$. However, we only need (a) of Theorem 4 to prove Theorem 2 
2.2. Proof of Theorem 3 (using Theorem 4). Let $r, \epsilon$, and $C>2$ be given, and set $\epsilon_{1}$ so that $C=2 /\left(1-2 \epsilon_{1}\right)$, (which implies $\left.\epsilon_{1}<1 / 2\right)$. Let $\delta_{1}=2 \epsilon /(r+2)$, and $t_{0}=t_{0}\left(r, \epsilon_{1}, \delta_{1}\right)$ for $r \geq 1$ or $t_{0}=t_{0}\left(\epsilon, \epsilon_{1}, \delta_{1}\right)$ for $r=0$ be determined by Theorem 4 . Let $\mathcal{A}$ and $\mathcal{B}$ be families, as in the setup of Theorem 3 , that satisfy (5). Then (9) holds, and we can apply Theorem 4 to get $\left(s, s^{\prime}\right) \in R_{\text {ex }}$ for which (a)-(c) hold. We consider the following three cases separately: Case $1\left(s, s^{\prime}\right)=(r+1, r)$ or $(r, r-1)$, Case $2\left(s, s^{\prime}\right)=(r+1, r+1)$ or $(r-1, r-1)$, Case $3\left(s, s^{\prime}\right)=(r, r)$.

- Case 1. Since $s=s^{\prime}+1$, we have $u=t-1$ and $v=t+1$. By (c) of Theorem 4 we have

$$
\mu_{p}(\mathcal{A}) \mu_{p}(\mathcal{B}) \leq \mu_{p}\left(\mathcal{F}_{s}^{t-1}\right) \mu_{p}\left(\mathcal{F}_{s^{\prime}}^{t+1}\right)
$$

Claim 2. Let $\left(s, s^{\prime}\right) \in\{(r+1, r),(r, r-1)\}$ and $0<\epsilon<1 / 2$. If $\frac{r+\epsilon}{t} \leq p \leq \frac{r+1-\epsilon}{t}$, then

$$
\frac{\mu_{p}\left(\mathcal{F}_{s}^{t-1}\right) \mu_{p}\left(\mathcal{F}_{s^{\prime}}^{t+1}\right)}{\mu_{p}\left(\mathcal{F}_{r}^{t}\right)^{2}} \leq\left(1-\frac{\epsilon}{r+1}\right)(1+o(1))
$$

Proof. Recall that $u=t-1$ and $v=t+1$. First, let $\left(s, s^{\prime}\right)=(r+1, r)$. Note that $r+\epsilon<t p<r+1-\epsilon$. Using (10), and in the last inequality that $t p<r+1-\epsilon$, we get

$$
\begin{aligned}
\frac{\mu_{p}\left(\mathcal{F}_{s}^{u}\right) \mu_{p}\left(\mathcal{F}_{s^{\prime}}^{v}\right)}{\mu_{p}\left(\mathcal{F}_{r}^{t}\right)^{2}} & =\frac{\left(\begin{array}{c}
t+2 r+1 \\
r+1
\end{array}\right) p^{t+r} q^{r+1}\left(\begin{array}{c}
t+2 r+1 \\
r
\end{array}\right) p^{t+r+1} q^{r}}{\left(\left(\begin{array}{c}
t+2 r \\
r
\end{array}\right) p^{t+r} q^{r}\right)^{2}}(1+o(1)) \\
& =\frac{(t+2 r+1)^{2} p q}{(r+1)(t+r+1)}(1+o(1))=\frac{t p q}{r+1}(1+o(1)) \\
& \leq\left(1-\frac{\epsilon}{r+1}\right)(1+o(1)) .
\end{aligned}
$$

Next, for the case $\left(s, s^{\prime}\right)=(r, r-1)$ one can similarly check that

$$
\begin{aligned}
\frac{\mu_{p}\left(\mathcal{F}_{s}^{u}\right) \mu_{p}\left(\mathcal{F}_{s^{\prime}}^{v}\right)}{\mu_{p}\left(\mathcal{F}_{r}^{t}\right)^{2}} & =\frac{\left(\begin{array}{c}
t+2 r-1 \\
r
\end{array}\right) p^{t+r-1} q^{r}\left(\begin{array}{c}
t+2 r-1 \\
r-1
\end{array}\right) p^{t+r} q^{r-1}}{\left(\left(\begin{array}{c}
t+2 r \\
r
\end{array}\right) p^{t+r} q^{r}\right)^{2}}(1+o(1)) \\
& =\frac{(t+r) r}{(t+2 r)^{2} p q}(1+o(1))=\frac{r}{t p q}(1+o(1)) \\
& \leq \frac{r}{r+\epsilon}(1+o(1))<1-\frac{\epsilon}{r+1} .
\end{aligned}
$$

Combining (11) and (12) contradicts (5), and hence Case 1 cannot happen.

- Case 2. Part (c) of Theorem 4 implies that

$$
\mu_{p}(\mathcal{A}) \mu_{p}(\mathcal{B}) \leq \mu_{p}\left(\mathcal{F}_{s}^{t}\right)^{2} .
$$

Claim 3. Let $0<\epsilon<1 / 2$. If $\frac{r+\epsilon}{t} \leq p \leq \frac{r+1-\epsilon}{t}$, then

$$
\frac{\max \left\{\mu_{p}\left(\mathcal{F}_{r-1}^{t}\right), \mu_{p}\left(\mathcal{F}_{r+1}^{t}\right)\right\}}{\mu_{p}\left(\mathcal{F}_{r}^{t}\right)} \leq\left(1-\frac{\epsilon}{r+1}\right)(1+o(1)) .
$$

Proof. Here we have $u=v=t$. By 10$]$ we get

$$
\begin{aligned}
\frac{\mu_{p}\left(\mathcal{F}_{r-1}^{t}\right)}{\mu_{p}\left(\mathcal{F}_{r}^{t}\right)} & =\frac{\left(\begin{array}{c}
t+2 r-2 \\
r-1
\end{array}\right) p^{t+r-1} q^{r-1}}{\left(\begin{array}{c}
t+2 r \\
r
\end{array}\right) p^{t+r} q^{r}}(1+o(1))=\frac{r(t+r)}{(t+2 r)(t+2 r-1) p q}(1+o(1)) \\
& =\frac{r(1+o(1))}{t p q} \leq \frac{r(1+o(1))}{r+\epsilon}<1-\frac{\epsilon}{r+1}
\end{aligned}
$$


and

$$
\begin{aligned}
\frac{\mu_{p}\left(\mathcal{F}_{r+1}^{t}\right)}{\mu_{p}\left(\mathcal{F}_{r}^{t}\right)} & =\frac{\left(\begin{array}{c}
t+2 r+2 \\
r+1
\end{array}\right) p^{t+r+1} q^{r+1}}{\left(\begin{array}{c}
t+2 r \\
r
\end{array}\right) p^{t+r} q^{r}}(1+o(1))=\frac{(t+2 r+2)(t+2 r+1) p q}{(r+1)(t+r+1)}(1+o(1)) \\
& =\frac{t p q(1+o(1))}{r+1} \leq\left(1-\frac{\epsilon}{r+1}\right)(1+o(1)) .
\end{aligned}
$$

Combining (13) and (14) again contradicts (5), and hence Case 2 cannot happen.

- Case 3. By the AM-GM inequality, and (5), we have

$$
X \geq 2 \sqrt{\mu_{p}(\mathcal{A}) \mu_{p}(\mathcal{B})} \geq 2 \sqrt{1-\delta} \mu\left(\mathcal{F}_{r}^{t}\right) .
$$

Recalling $X, X_{\mathcal{F}}, X_{\Delta}, X_{*}$ from the definition preceding Theorem 4 an inclusionexclusion argument gives that

$$
\begin{aligned}
X_{\Delta} & =X_{\mathcal{F}}+2 X_{*}-X \leq 2 \mu_{p}\left(\mathcal{F}_{r}^{t}\right)+2 X_{*}-2 \sqrt{1-\delta} \mu_{p}\left(\mathcal{F}_{r}^{t}\right) \\
& =2(1-\sqrt{1-\delta}) \mu_{p}\left(\mathcal{F}_{r}^{t}\right)+2 X_{*} .
\end{aligned}
$$

This together with $X_{*} \leq \epsilon_{1} X_{\Delta}$ from (b) of Theorem 4 yields

$$
X_{\Delta} \leq 2(1-\sqrt{1-\delta}) \mu_{p}\left(\mathcal{F}_{r}^{t}\right)+2 \epsilon_{1} X_{\Delta} .
$$

Since $0<\epsilon_{1}<1 / 2$ we have

$$
X_{\Delta} \leq \frac{2(1-\sqrt{1-\delta})}{1-2 \epsilon_{1}} \mu_{p}\left(\mathcal{F}_{r}^{t}\right)=C(1-\sqrt{1-\delta}) \mu_{p}\left(\mathcal{F}_{r}^{t}\right) .
$$

This proves (6), and completes the proof of Theorem 3

\section{Preliminary tools}

3.1. Walks corresponding to subsets. It is useful to regard a set $F \subset[n]$ as an $n$-step walk starting at the origin $(0,0)$ of the two-dimensional grid $\mathbb{Z}^{2}$ as follows. If $i \in F$, then the $i$-th step is up from $(x, y)$ to $(x, y+1)$. Otherwise, the $i$-th step is right from $(x, y)$ to $(x+1, y)$. From now on, we refer to $F \subset[n]$ as a set or a walk.

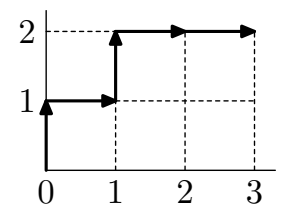

FiguRE 1. Walk corresponding to $\{1,3\} \subset[5]$

A walk $F$ reaches the point $(j, j+\ell)$ on the line $y=x+\ell$ if and only if $|F \cap[\ell+2 j]|=$ $\ell+j$. For an integer $\ell \geq 0$ let

$$
\mathcal{F}^{\ell}:=\bigcup_{j \geq 0} \mathcal{F}_{j}^{\ell}=\{F \subset[n]:|F \cap[\ell+2 j]| \geq \ell+j \text { for some } j \geq 0\}
$$

be the family of all walks that hit the line $y=x+\ell$, where $\mathcal{F}_{j}^{\ell}$ is defined in (1). 
We further define

$\tilde{\mathcal{F}}^{\ell}:=\mathcal{F}^{\ell+1}=\left\{F \in \mathcal{F}^{\ell}: F\right.$ hits $\left.y=x+\ell+1\right\}$,

$\dot{\mathcal{F}}^{\ell}:=\left\{F \in \mathcal{F}^{\ell}: F\right.$ hits $y=x+\ell$ exactly once, but does not hit $\left.y=x+\ell+1\right\}$,

$\ddot{\mathcal{F}}^{\ell}:=\left\{F \in \mathcal{F}^{\ell}: F\right.$ hits $y=x+\ell$ at least twice, but does not hit $\left.y=x+\ell+1\right\}$.

This gives a partition $\mathcal{F}^{\ell}=\tilde{\mathcal{F}}^{\ell} \sqcup \dot{\mathcal{F}}^{\ell} \sqcup \ddot{\mathcal{F}}^{\ell}$. Let $\dot{\mathcal{F}}_{i}^{\ell}:=\dot{\mathcal{F}}^{\ell} \cap \mathcal{F}_{i}^{\ell}$ and $\ddot{\mathcal{F}}_{i}^{\ell}:=\ddot{\mathcal{F}}^{\ell} \cap \mathcal{F}_{i}^{\ell}$.

One can estimate the measure of the families defined above using random walks, and this is one of the main ideas for proving our results. Consider an infinite random walk in the plane starting from the origin, each step of which is a random variable, independent of other steps, going up with probability $p$ and right with probability $q:=1-p$. The product measure of the family of walks that satisfy some property is the probability of a random walk satisfying that property.

Example 4. Let $\mathcal{F}$ be the family of all walks that hit the point $(s, u+s)$ for integers $u$ and $s$. There are $\left(\begin{array}{c}u+2 s \\ s\end{array}\right)$ different walks from $(0,0)$ to $(s, u+s)$, and a random walk is any one of these with probability $p^{u+s} q^{s}$, so $\mu_{p}(\mathcal{F})=\left(\begin{array}{c}u+2 s \\ s\end{array}\right) p^{u+s} q^{s}$.

With a little more work, one can show that the infinite random walk hits the line $y=x+\ell$ with probability precisely $\alpha^{\ell}$, where $\alpha:=p / q$. Based on this fact, one can show the following.

Lemma 5 (Lemma 2.2 in [13]). For any positive integer $\ell$, the following hold.

(i) We have $\mu_{p}\left(\mathcal{F}^{\ell}\right) \leq \alpha^{\ell}, \mu_{p}\left(\tilde{\mathcal{F}}^{\ell}\right) \leq \alpha^{\ell+1}$, and $\mu_{p}\left(\ddot{\mathcal{F}}^{\ell}\right) \leq \alpha^{\ell+1}$.

(ii) If $\mathcal{F} \subset 2^{[n]}$ and no walk in $\mathcal{F}$ hits the line $y=x+\ell$, then $\mu_{p}(\mathcal{F})<1-\alpha^{\ell}+o(1)$, where $o(1) \rightarrow 0$ as $n \rightarrow \infty$.

We will also use the following fact.

Fact 6 (Lemma 2.13 in [13]). The number of walks from $(0,0)$ to $(s, \ell+s) \in \mathbb{N}^{2}$ that do not hit the line $y=x+\ell+1$ is $\left(\begin{array}{c}\ell+2 s \\ s\end{array}\right)-\left(\begin{array}{c}\ell+2 s \\ s-1\end{array}\right)$.

3.2. Dual walks and some facts. For $A \subset[n]$, let $(A)_{i}$ be the $i$-th smallest element of $A$. For $A, B \subset[n]$, we say that $A$ shifts to $B$, denoted by $A \rightarrow B$, if $|A| \leq|B|$ and $(A)_{i} \geq(B)_{i}$ for each $i \leq|A|$. As an example, we have $\{2,4,6\} \rightarrow\{1,4,5,7\}$. Viewing $A$ and $B$ as walks, $A \rightarrow B$ means that the walk $B$ is above the walk $A$ on the grid $\mathbb{Z}^{2}$. The dual of $A \subset[n]$ with respect to $t$ is defined by

$$
\operatorname{dual}_{t}(A):=\left[(A)_{t}-1\right] \cup([n] \backslash A) .
$$

See Figure 3 for an example of a walk and its dual.

Note that $\left|A \cap \operatorname{dual}_{t}(A)\right|=t-1$. Furthermore, $\operatorname{dual}_{t}(A)$ is the shift minimal walk satisfying this condition, and hence, if $|A \cap B|=t-1$ then $B \rightarrow \operatorname{dual}_{t}(A)$. As walks, $\operatorname{dual}_{t}(A)$ is obtained by reflecting $A$ across the line $y=x+(t-1)$ and replacing the part $x<0$ with the path connecting $(0,0)$ and $\left(0,(A)_{t}-1\right)$. The following fact is immediate.

Fact 7 (Facts 2.8 and 2.9 in [13]).

(i) Let $\mathcal{F}$ be a shifted, inclusion maximal family in $2^{[n]}$. If $F \in \mathcal{F}$ and $F \rightarrow F^{\prime}$, then $F^{\prime} \in \mathcal{F}$.

(ii) Let $\mathcal{A}$ and $\mathcal{B}$ be cross $t$-intersecting families. If $A \in \mathcal{A}$, then dual ${ }_{t}(A) \notin \mathcal{B}$. 


\section{Setup for the Proof of Theorem 4}

The rest of the paper is dedicated to the proof of Theorem 4

4.1. Basic Asymptotics. In this section we talk about the assumptions we will use in asymptotic arguments. From now on, we let $r \geq 0$ be a fixed integer, and if $r=0$ then let $\epsilon>0$ also be fixed. Let let $t$ be a sufficiently large integer depending on $r$ and epsilon. Since we are interested in the maximum possible measure $\mu_{p}(\mathcal{A}) \mu_{p}(\mathcal{B})$ over all $t$-nice pairs $\{\mathcal{A}, \mathcal{B}\}$, where $\mathcal{A}, \mathcal{B} \subset 2^{[n]}$, and this value is non-decreasing in $n$ (see Lemma 2.12 in [13]), we may assume that $n$ is sufficiently large compared to $t$. Consequently, we assume that

$$
0 \leq r \ll t \ll n .
$$

First assume that $r \geq 1$, and $p$ satisfies

which gives

$$
\frac{r}{t+2 r-1} \leq p \leq \frac{r+1}{t+2 r+1}
$$

$$
p=\Theta(1 / t)=o(1) \text { and } r-o(1)<t p<r+1,
$$

where $o(1)$ goes to 0 as $t \rightarrow \infty$. This implies that $\left(1-\frac{r+1}{t}\right)^{t}<(1-p)^{t}<\left(1-\frac{r-o(1)}{t}\right)^{t}$, and using $q=1-p$ it follows that $e^{-(r+1)}(1-o(1))<q^{t}<e^{-r}(1+o(1))$; in particular, $q^{t}=\Theta(1)$. We also have that

$$
\alpha=\frac{p}{q}=p+p^{2}+\cdots=p+O\left(p^{2}\right) \quad \text { and } \quad \alpha^{t}=\frac{p^{t}}{q^{t}}=\Theta\left(p^{t}\right) .
$$

To simplify (1) we write

$\mu_{p}\left(\mathcal{F}_{r}^{t}\right)=\sum_{i=0}^{r}\left(\begin{array}{c}t+2 r \\ i\end{array}\right) p^{t+2 r-i} q^{i}=\left(\begin{array}{c}t+2 r \\ r\end{array}\right) p^{t+r} q^{r}\left(1+\Theta\left(1 / t^{2}\right)\right)=\frac{(t p)^{r}}{r !} p^{t}\left(1+\Theta\left(1 / t^{2}\right)\right)$.

Using 17], this reads

$$
\mu_{p}\left(\mathcal{F}_{r}^{t}\right)=\Theta\left(p^{t}\right)
$$

Suppose now that $r=0$ and that $p>\epsilon / t$. Then (18) and (20) trivially hold. In particular, $\mu_{p}(\mathcal{A}) \mu_{p}(\mathcal{B})=\Theta\left(p^{2 t}\right)$ holds for any pair $\{\mathcal{A}, \mathcal{B}\}$ in (7) for $r \geq 1$, as well as $\{\mathcal{A}, \mathcal{B}\}$ in $\left\{\mathcal{F}_{0}^{t}, \mathcal{F}_{0}^{t}\right\},\left\{\mathcal{F}_{1}^{t}, \mathcal{F}_{1}^{t}\right\}$, and $\left\{\mathcal{F}_{1}^{t-1}, \mathcal{F}_{0}^{t}\right\}$.

4.2. Initial reductions and definition of cases. In this section, we make initial reductions for the proof of Theorem 4, and introduce parameters by which we can break the remainder of the proof into cases.

We have already fixed $r \geq 0$. Let $\epsilon, \epsilon_{1} \in(0,1 / 2)$ and $\delta_{1} \in(0,1 /(r+2))$ be given. We will choose $t_{0}$, depending on these constants, to be sufficiently large. Only $\delta_{1}$ matters in this and the next sections, and then $\epsilon$ or $\epsilon_{1}$ will get involved in Sections 6 and 7. Choose $n \gg t \geq t_{0}$.

Let $\mathcal{A}, \mathcal{B} \subset 2^{[n]}$ be $t$-nice families. Where $\lambda(\mathcal{F})$, for $\mathcal{F} \subset 2^{[n]}$, is the maximum $\ell$ such that $\mathcal{F} \subset \mathcal{F}^{\ell}$, let

$$
u:=\lambda(\mathcal{A}) \text { and } v:=\lambda(\mathcal{B}) .
$$

Without loss of generality, we may assume that $u \leq v$. Since $\mathcal{A}$ and $\mathcal{B}$ are $t$-nice, we have $u+v \geq 2 t$, see e.g., Lemma 2.11 of $[13$.

Since $\mathcal{A} \subset \mathcal{F}^{u}$, we have a partition $\mathcal{A}=\tilde{\mathcal{A}} \sqcup \dot{\mathcal{A}} \sqcup \ddot{\mathcal{A}}$, where $\tilde{\mathcal{A}}=\mathcal{A} \cap \tilde{\mathcal{F}}^{u}, \dot{\mathcal{A}}=\mathcal{A} \cap \dot{\mathcal{F}}^{u}$, and $\ddot{\mathcal{A}}=\mathcal{A} \cap \ddot{\mathcal{F}}^{u}$. Similarly, we have a partition $\mathcal{B}=\tilde{\mathcal{B}} \sqcup \dot{\mathcal{B}} \sqcup \ddot{\mathcal{B}} \subset \mathcal{F}^{v}$. 
Lemma 8. If either $u+v>2 t, \dot{\mathcal{A}}=\emptyset$, or $\dot{\mathcal{B}}=\emptyset$, then $\mu_{p}(\mathcal{A}) \mu_{p}(\mathcal{B}) \ll \mu_{p}\left(\mathcal{F}_{r}^{t}\right)^{2}$.

Proof. Suppose that $u+v>2 t$. Lemma 5 yields that $\mu_{p}(\mathcal{A}) \leq \alpha^{u}$ and $\mu_{p}(\mathcal{B}) \leq \alpha^{v}$, so by (18) and (20) it follows $\mu_{p}(\mathcal{A}) \mu_{p}(\mathcal{B}) \leq \alpha^{u} \alpha^{v} \leq \alpha^{2 t+1}=O\left(p^{2 t+1}\right) \ll \mu_{p}\left(\mathcal{F}_{r}^{t}\right)^{2}$.

Next suppose that $\dot{\mathcal{A}}=\emptyset$, then Lemma 5 gives $\mu_{p}(\mathcal{A})=\mu_{p}(\tilde{\mathcal{A}})+\mu_{p}(\ddot{\mathcal{A}}) \leq 2 \alpha^{u+1}$, and thus $\mu_{p}(\mathcal{A}) \mu_{p}(\mathcal{B}) \leq 2 \alpha^{u+1} \alpha^{v}=2 \alpha^{2 t+1} \ll \mu_{p}\left(\mathcal{F}_{r}^{t}\right)^{2}$. The same holds if $\dot{\mathcal{B}}=\emptyset$.

Lemma 8 guarantees the existence of $t_{0}$ depending on $r$ and $\delta_{1}$ such that if $t \geq t_{0}$ and $\mathcal{A}$ and $\mathcal{B}$ are $t$-nice families satisfying (9), then we necessarily have that $u+v=2 t$, $\dot{\mathcal{A}} \neq \emptyset$, and $\dot{\mathcal{B}} \neq \emptyset$. Moreover, one can show the following.

Lemma 9 (Lemma 3.2 in 13 ). Suppose that $\dot{\mathcal{A}} \neq \emptyset$ and $\dot{\mathcal{B}} \neq \emptyset$. Then, there exist unique non-negative integers $s$ and $s^{\prime}$ such that $\dot{\mathcal{A}} \sqcup \ddot{\mathcal{A}} \subset \mathcal{F}_{s}^{u}$ and $\dot{\mathcal{B}} \sqcup \ddot{\mathcal{B}} \subset \mathcal{F}_{s^{\prime}}^{v}$. Moreover, $s-s^{\prime}=(v-u) / 2$. In particular, $s \geq s^{\prime}$.

Here, we record the main discussion of this and the previous section.

Setup. For a proof of Theorem 4 we may assume the following.

- $r \geq 0$ is a fixed integer.

- $\epsilon, \epsilon_{1} \in(0,1 / 2)$, and $\delta_{1} \in\left(0, \frac{1}{r+2}\right)$ are fixed real numbers.

- $r \ll t \ll n$. ( $t$ depends on $r, \epsilon, \epsilon_{1}, \delta_{1}$, which will be described later.)

- $\frac{r}{t+2 r-1} \leq p \leq \frac{r+1}{t+2 r+1}$ for $r \geq 1$, and $\frac{\epsilon}{t} \leq p \leq \frac{1}{t+1}$ for $r=0$.

- $q=1-p$ and $\alpha=p / q$.

- $\mathcal{A}, \mathcal{B} \subset 2^{[n]}$ are $t$-nice, that is, shifted, inclusion maximal, and cross $t$ intersecting.

- $u=\lambda(\mathcal{A}), v=\lambda(\mathcal{B}), u+v=2 t, 1 \leq u \leq t \leq v \leq 2 t$.

- $s \geq s^{\prime} \geq 0, s-s^{\prime}=(v-u) / 2, u=t-\left(s-s^{\prime}\right), v=t+s-s^{\prime}$.

- $\mathcal{A}=\tilde{\mathcal{A}} \sqcup \dot{\mathcal{A}} \sqcup \ddot{\mathcal{A}} \subset \mathcal{F}^{u}, \mathcal{B}=\tilde{\mathcal{B}} \sqcup \dot{\mathcal{B}} \sqcup \ddot{\mathcal{B}} \subset \mathcal{F}^{v}, \dot{\mathcal{A}} \neq \emptyset, \dot{\mathcal{B}} \neq \emptyset, \dot{\mathcal{A}} \sqcup \ddot{\mathcal{A}} \subset \mathcal{F}_{s}^{u}$, $\dot{\mathcal{B}} \sqcup \ddot{\mathcal{B}} \subset \mathcal{F}_{s^{\prime}}^{v}$

Under this setup, the proof of Theorem 4 breaks down into three cases: Recalling $R_{\text {ex }}$ defined in (8), we consider NE: the non-extremal case $\left(s, s^{\prime}\right) \notin R_{\text {ex }}$, DE: the diagonal extremal case $\left(s, s^{\prime}\right) \in R_{\text {ex }}$ and $s=s^{\prime}$, NDE: the non-diagonal extremal case $\left(s, s^{\prime}\right) \in R_{\text {ex }}$ and $s=s^{\prime}+1$.

\section{NON-EXTREMAL CASES}

In this section, we deal with the case $\mathrm{NE}$, that is, the case when $\left(s, s^{\prime}\right) \notin R_{\mathrm{ex}}$. We prove the following lemma. By choosing $t_{0}$, depending on $\delta_{1}$, sufficiently large, the lemma shows that Theorem 4 holds vacuously for $t \geq t_{0}$, since $(9)$ does not hold.

Lemma 10. If $\left(s, s^{\prime}\right) \notin R_{\text {ex }}$ then $\mu_{p}(\mathcal{A}) \mu_{p}(\mathcal{B})<\left(1-\frac{1}{r+2}\right) \mu_{p}\left(\mathcal{F}_{r}^{t}\right)^{2}(1+o(1))$.

For a proof of Lemma 10 recall that $\mathcal{A}=\tilde{\mathcal{A}} \sqcup \dot{\mathcal{A}} \sqcup \ddot{\mathcal{A}} \subset \tilde{\mathcal{F}}^{u} \sqcup \dot{\mathcal{F}}_{s}^{u} \sqcup \ddot{\mathcal{F}}_{s}^{u}$. Recall also that no walk in $\dot{\mathcal{F}}_{s}^{u} \sqcup \ddot{\mathcal{F}}_{s}^{u}$ hits the line $y=x+u+1$ while all walks in $\mathcal{F}_{s}^{u}$ hit one of $(0, u+2 s),(1, u+2 s-1), \ldots,(s, u+s)$. Since $\dot{\mathcal{F}}_{s}^{u} \sqcup \ddot{\mathcal{F}}_{s}^{u} \subset \mathcal{F}_{s}^{u}$ all walks in $\dot{\mathcal{F}}_{s}^{u} \sqcup \ddot{\mathcal{F}}_{s}^{u}$ must hit $(s, u+s)$. Thus, by Example 4 , we have

$$
\mu_{p}\left(\dot{\mathcal{F}}_{s}^{u} \sqcup \ddot{\mathcal{F}}_{s}^{u}\right) \leq\left(\begin{array}{c}
u+2 s \\
s
\end{array}\right) p^{u+s} q^{s}<\left(\begin{array}{c}
u+2 s \\
s
\end{array}\right) p^{u+s} .
$$


This together with $\mu_{p}\left(\tilde{\mathcal{F}}^{u}\right) \leq \alpha^{u+1}=(p / q)^{u+1}$, which we get from Lemma 5 yields

$$
\mu_{p}(\mathcal{A}) \leq \mu_{p}\left(\tilde{\mathcal{F}}^{u} \sqcup \dot{\mathcal{F}}_{s}^{u} \sqcup \ddot{\mathcal{F}}_{s}^{u}\right)<\left(\frac{p}{q}\right)^{u+1}+\left(\begin{array}{c}
u+2 s \\
s
\end{array}\right) p^{u+s}=h(u, s) p^{u},
$$

where

$$
h(i, j):=\frac{p}{q^{i+1}}+\left(\begin{array}{c}
i+2 j \\
j
\end{array}\right) p^{j} .
$$

Similarly we have $\mu_{p}(\mathcal{B}) \leq h\left(v, s^{\prime}\right) p^{v}$. Thus we have

$$
\mu_{p}(\mathcal{A}) \mu_{p}(\mathcal{B})<h(u, v) h\left(v, s^{\prime}\right) p^{u+v}=h(u, v) h\left(v, s^{\prime}\right) p^{2 t} .
$$

Hence, in order to show Lemma 10, it suffices to show

$$
h(u, s) h\left(v, s^{\prime}\right) p^{2 t}<\frac{r+1}{r+2} \mu_{p}\left(\mathcal{F}_{r}^{t}\right)^{2}(1+o(1)) .
$$

5.1. The case when $s$ is large. In this subsection we show 22 for the case $s \geq$ $2 e(r+1)$. We start by bounding the terms in $h(u, s)$ and $h\left(v, s^{\prime}\right)$ using the following claim, which uses only elementary calculus.

Claim 11. If $s \geq 2 e(r+1)$, then we have

(i) $\max \left\{p / q^{u+1}, p / q^{v+1}\right\}=O(p)$,

(ii) $\left(\begin{array}{c}u+2 s \\ s\end{array}\right) p^{s}<e^{-e(r+1)}$,

(iii) $\left(\begin{array}{c}v+2 s^{\prime} \\ s^{\prime}\end{array}\right) p^{s^{\prime}}<1.001 e^{2(r+1)}$.

Proof. (i) Using $u \leq v \leq 2 t$ and $q^{t}=\Theta(1)$, we get $\max \left\{p / q^{u+1}, p / q^{v+1}\right\} \leq p / q^{2 t+1}=$ $O(p)$.

(ii) We have $\left(\begin{array}{c}u+2 s \\ s\end{array}\right) p^{s}<\frac{(u+2 s)^{s}}{s !} p^{s}<\frac{(u+2 s)^{s}}{(s / e)^{s}} p^{s}=\left(\frac{e u p}{s}+2 e p\right)^{s}$. Using $u p \leq t p<$ $r+1$ and the assumption $s \geq 2 e(r+1)$, we have $($ eup $) / s<1 / 2$. Also, we have $2 e p<0.1$. Thus we get $\left(\begin{array}{c}u+2 s \\ s\end{array}\right) p^{s}<\left(\frac{1}{2}+0.1\right)^{s}<\left(\frac{1}{\sqrt{e}}\right)^{s} \leq\left(\frac{1}{\sqrt{e}}\right)^{2 e(r+1)}=e^{-e(r+1)}$.

(iii) If $s^{\prime}=0$ then the inequality is true. Let $s^{\prime}>0$. First observe

$$
\begin{aligned}
\left(\begin{array}{c}
v+2 s^{\prime} \\
s^{\prime}
\end{array}\right) p^{s^{\prime}} & <\left(\begin{array}{c}
2 t+2 s^{\prime} \\
s^{\prime}
\end{array}\right)\left(\frac{r+1}{t}\right)^{s^{\prime}}<\frac{\left(2 t+2 s^{\prime}\right)^{s^{\prime}}}{\left(s^{\prime} / e\right)^{s^{\prime}}}\left(\frac{r+1}{t}\right)^{s^{\prime}} \\
& =\left(\frac{2 e(r+1)\left(s^{\prime}+t\right)}{s^{\prime} t}\right)^{s^{\prime}}=: \gamma .
\end{aligned}
$$

We consider now three cases, depending on $s^{\prime}$.

- If $s^{\prime} \geq \sqrt{t}$ then $\frac{s^{\prime}+t}{s^{\prime} t} \leq \frac{\sqrt{t}+t}{\sqrt{t} t}=\frac{1}{t}+\frac{1}{\sqrt{t}}<\frac{1}{2 e(r+1)}$ for $t \geq t_{0}$. Thus $\gamma<1$.

- If $2 e(r+1)<s^{\prime}<\sqrt{t}$ then $\frac{2 e(r+1)}{s^{\prime}}<1$, and hence, $\gamma<\left(\frac{s^{\prime}+t}{t}\right)^{s^{\prime}}<$ $\left(\frac{t+\sqrt{t}}{t}\right)^{\sqrt{t}}<e$.

- Finally let $0<s^{\prime}<2 e(r+1)$. We divide $\gamma$ into two parts $\left(\frac{s^{\prime}+t}{t}\right)^{s^{\prime}}$ and $\left(\frac{2 e(r+1)}{s^{\prime}}\right)^{s^{\prime}}$. For the first part we have $\left(\frac{s^{\prime}+t}{t}\right)^{s^{\prime}}<\left(1+\frac{2 e(r+1)}{t}\right)^{2 e(r+1)}<$ 1.001 for $t \geq t_{0}$. For the second part, the derivative gives that $\left(\frac{2 e(r+1)}{s^{\prime}}\right)^{s^{\prime}}$ is maximized at $s^{\prime}=2(r+1)$ as $e^{2(r+1)}$. Thus $\gamma<1.001 e^{2(r+1)}$.

This completes the proof of the claim. 
Now we prove 22 . By the previous claim we have

$$
\begin{aligned}
h(u, s) h\left(v, s^{\prime}\right) & <\left(O(p)+e^{-e(r+1)}\right)\left(O(p)+1.001 e^{2(r+1)}\right) \\
& =1.001 e^{-(r+1)(e-2)}+O(p)<1.01 e^{-(r+1)(e-2)} .
\end{aligned}
$$

Using $\mu_{p}\left(\mathcal{F}_{r}^{t}\right) \geq \mu_{p}\left(\mathcal{F}_{0}^{t}\right)=p^{t}$ for $r \geq 1$ we get

$$
\frac{h(u, s) h\left(v, s^{\prime}\right) p^{2 t}}{\mu_{p}\left(\mathcal{F}_{r}^{t}\right)^{2}} \leq h(u, s) h\left(v, s^{\prime}\right)<1.01 e^{-2(e-2)}<\frac{1}{4}<\frac{r+1}{r+2} .
$$

This completes the proof of $(22)$, and thus, of Lemma 10 for the case $s \geq 2 e(r+1)$.

5.2. The case when $s$ is small. In this subsection we show 22 for the case $s<$ $2 e(r+1)$. In this case, we take advantage of the fact that $s=O(1)$ is much smaller than $t$. Let us estimate $h(u, s)$ defined in (21). Using $p / q^{u+1}=o(1)$ and $u+2 s \leq$ $t+2 s=t+O(1)$ we have

$$
h(u, s)=\frac{p}{q^{u+1}}+\left(\begin{array}{c}
u+2 s \\
s
\end{array}\right) p^{s} \leq o(1)+\frac{(t+O(1))^{s}}{s !} p^{s}=\frac{(t p)^{s}}{s !}(1+o(1)) .
$$

Similarly, $h\left(v, s^{\prime}\right)=\frac{\left(t p s^{s^{\prime}}\right.}{s^{\prime} !}(1+o(1))$. Combining these with $\mu_{p}\left(\mathcal{F}_{r}^{t}\right)=\frac{(t p)^{r}}{r !} p^{t}(1+o(1))$ (cf. (19p), we have

$$
g\left(s, s^{\prime}\right):=\frac{h(u, s) h\left(v, s^{\prime}\right) p^{2 t}}{\mu_{p}\left(\mathcal{F}_{r}^{t}\right)^{2}}=\frac{(r !)^{2}(t p)^{s+s^{\prime}-2 r}}{s ! s^{\prime} !}(1+o(1)) .
$$

In order to prove $(22)$, it suffices to show that

$$
g\left(s, s^{\prime}\right)<\frac{r+1}{r+2}(1+o(1)) .
$$

Our proof of $(24)$ is based on the following observation.

Claim 12. Let $S=\{s \in \mathbb{N}: 0 \leq s<2 e(r+1)\}$. There is $t_{0}$ depending on $r$ such that for $t \geq t_{0}$ the following holds.

(i) $g\left(s, s^{\prime}\right)>g\left(s+1, s^{\prime}-1\right)$ for $s \geq s^{\prime}>0$.

(ii) $g(s, s) \leq \max \{g(r-2, r-2), g(r+2, r+2)\}$ for $s \in S \backslash\{r-1, r, r+1\}$,

(iii) $g(s, s-1) \leq \max \{g(r-1, r-2), g(r+2, r+1)\}$ for $s \in S \backslash\{0, r, r+1\}$.

Of course, $s$ and $s^{\prime}$ can never be negative, so when $r=0$ or 1 we replace $g(\cdot, r-2)$ in (ii) or (iii) with 0 .

Proof. (i) It follows from (23) that if $t$ is large enough then $g\left(s, s^{\prime}\right)>g\left(s+1, s^{\prime}-1\right)$ is equivalent to $s+1>s^{\prime}$.

(ii) Recall that $r-o(1)<t p<r+1$. By comparing $g(x-1, x-1)$ and $g(x, x)$, we have that $g(1,1)<g(2,2)<\cdots<g(r-1, r-1)$ and $g(r, r)>g(r+1, r+1)>\cdots$ if $t$ is large enough.

(iii) Let $F(s):=g(s, s-1)$. Then, by (23), we have that if $t$ is large enough then $F(s)<F(s+1)$ is equivalent to $(s+1) s<(t p)^{2}$. Hence, $F(1)<F(2)<\cdots<F(r)$ and $F(r+1)>F(r+2)>\cdots$. Indeed, if $s \leq r-1$, then $(s+1) s \leq r(r-1)<$ $(r-o(1))^{2}<(t p)^{2}$. On the other hand, if $s \geq r+1$, then $(s+1) s \geq(r+2)(r+1)>$ $(r+1)^{2}>(t p)^{2}$. 
The arrows in Figure 2 illustrates the relation between the values $g\left(s, s^{\prime}\right)$ for values of $s$ and $s^{\prime}$ considered in Claim 12. We mention that 24) does not hold for $\left(s, s^{\prime}\right) \in$ $R_{\mathrm{ex}}$, which is the reason that we do not draw arrows starting from the points in $R_{\mathrm{ex}}$. The figure tells us that in order to show 24 for $\left(s, s^{\prime}\right) \notin R_{\mathrm{ex}}$ it suffices to check the following starting points when $s$ and $s^{\prime}$ are non-negative:

$$
\begin{aligned}
\left(s, s^{\prime}\right) \in\{ & (r-2, r-2),(r+2, r+2),(r-1, r-2),(r+2, r+1), \\
& (r+2, r),(r+2, r-1),(r+1, r-1),(r+1, r-2),(r, r-2)\} .
\end{aligned}
$$

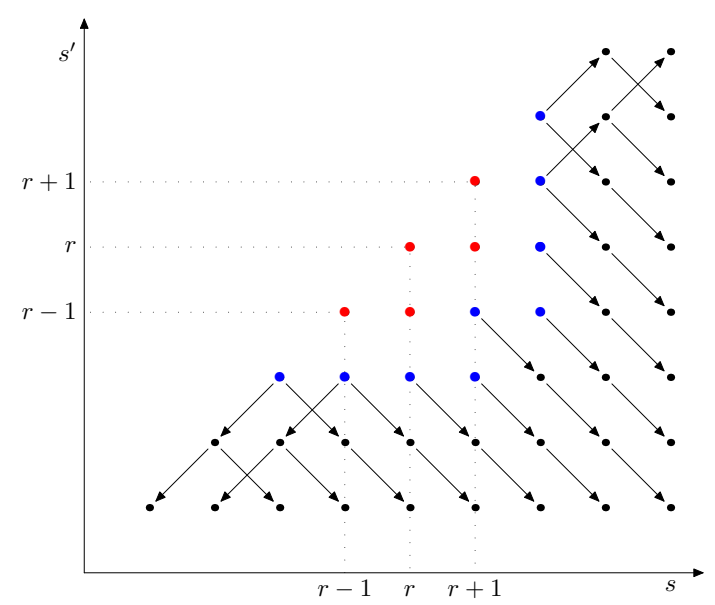

Figure 2. Relations on $g\left(s, s^{\prime}\right)$

The verification of (24) for these cases follows from easy computation. For example,

$$
g(r-2, r-2) \leq \frac{r^{2}(r-1)^{2}}{(t p)^{4}}(1+o(1)) \leq \frac{(r-1)^{2}}{r^{2}}(1+o(1)) \rightarrow \frac{(r-1)^{2}}{r^{2}}
$$

as $t \rightarrow \infty$, and to mean this situation we write $g(r-2, r-2) \nearrow \frac{(r-1)^{2}}{r^{2}}$. Similarly we have

$$
\begin{array}{lll}
g(r+2, r+2) \nearrow \frac{(r+1)^{2}}{(r+2)^{2}}, & g(r-1, r-2) \nearrow \frac{r-1}{r}, & g(r+2, r+1) \nearrow \frac{r+1}{r+2}, \\
g(r+2, r) \nearrow \frac{r+1}{r+2}, & g(r+2, r-1) \nearrow \frac{r}{r+2}, & g(r+1, r-1) \nearrow \frac{r}{r+1}, \\
g(r+1, r-2) \nearrow \frac{r-1}{r+1}, & g(r, r-2)\} \nearrow \frac{r-1}{r} .
\end{array}
$$

Therefore, we get $(24)$ for all $\left(s, s^{\prime}\right) \notin R_{\mathrm{ex}}$, which completes the proof of Lemma 10 for the case $s<2 e(r+1)$.

So far, we have proved Theorem 4 in the case ND.

\section{Diagonal extremal Cases}

In this section we deal with the case DE, that is, we assume that

$$
\left(s, s^{\prime}\right)=(r-1, r-1),(r, r), \text { or }(r+1, r+1),
$$




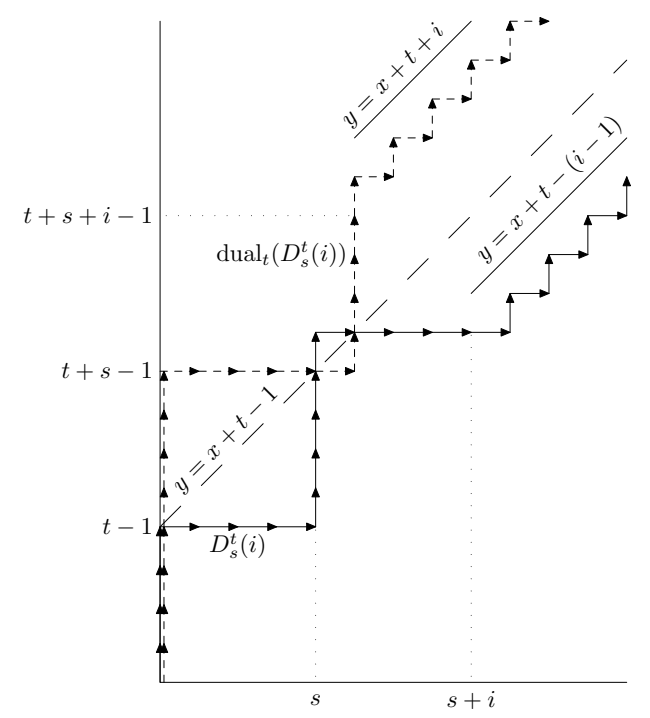

Figure 3. Walks $D_{s}^{t}(i)$ and $\operatorname{dual}_{t}\left(D_{s}^{t}(i)\right)$

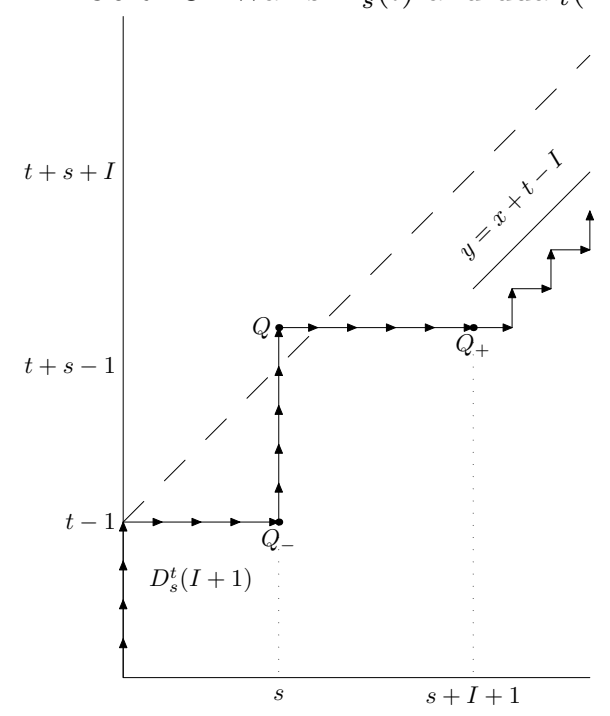

FiguRE 4. Walk $D_{s}^{t}(I+1)$

or the latter two only, if $r=0$. Under the assumption $s=s^{\prime}$ we have that $u=v=t$. Defining the notation $[a, b]_{2}:=\{a+2 i: i \in \mathbb{Z}\} \cap[a, b]$, we let

$$
D_{s}^{t}(i):=[t-1] \cup[t+s, t+2 s] \cup[t+2 s+i+2, n]_{2} \in \dot{\mathcal{F}}_{s}^{t} .
$$

The parameter $i$ ranges over $1 \leq i \leq n-t-2 s-1=: i_{\max }$, where $i_{\max }$ is defined so that $D_{s}^{t}\left(i_{\max }\right)=[t-1] \cup[t+s, t+2 s]$. Recalling the definition of the dual walk in (16) on page 9, we have (see Figure 3)

$$
\operatorname{dual}_{t}\left(D_{s}^{t}(i)\right)=[t+s-1] \cup[t+2 s+1, t+2 s+i+1] \cup[t+2 s+i+3, n]_{2}
$$


Consider the case where $D_{s}^{t}(1) \notin \mathcal{A}$ or $D_{s}^{t}(1) \notin \mathcal{B}$. By symmetry we may assume that $D_{s}^{t}(1) \notin \mathcal{A}$. In this case we show that Theorem 4 vacuously holds since $(9)$ does not hold.

Lemma 13. If $D_{s}^{t}(1) \notin \mathcal{A}$ then $\mu_{p}(\mathcal{A}) \mu_{p}(\mathcal{B}) \ll \mu_{p}\left(\mathcal{F}_{r}^{t}\right)^{2}$, that is, there exists $t_{0}$ depending on $r$ and $\delta_{1}$ such that $\mu_{p}(\mathcal{A}) \mu_{p}(\mathcal{B})<\left(1-\delta_{1}\right) \mu_{p}\left(\mathcal{F}_{r}^{t}\right)^{2}$ for all $t \geq t_{0}$.

Proof. First we show that

$$
\mu_{p}(\dot{\mathcal{A}})=O\left(p^{t+1}\right)
$$

For the proof, let $\mathcal{W}:=\left\{W \in \dot{\mathcal{F}}_{s}^{t}: W \rightarrow D_{s}^{t}(1)\right\}$. Since $D_{s}^{t}(1) \notin \mathcal{A}$, Fact 7 (i) gives that $W \rightarrow D_{s}^{t}(1)$ implies $W \notin \mathcal{A}$. As $\dot{\mathcal{A}} \subset \mathcal{F}_{s}^{t}$ and so $\dot{\mathcal{A}} \subset \dot{\mathcal{F}}_{s}^{t}$ we have $\dot{\mathcal{A}} \subset \dot{\mathcal{F}}_{s}^{t} \backslash \mathcal{W}$, and hence, $\mu_{p}(\dot{\mathcal{A}}) \leq \mu_{p}\left(\dot{\mathcal{F}}_{s}^{t} \backslash \mathcal{W}\right)$. Now, all walks in $\dot{\mathcal{F}}_{s}^{t}$ hit the line $y=x+t$ only at $(s, t+s)$. Hence, they necessarily hit $(s, t+s-1)$ and the $(t+2 s)$-th step is 'up'. Using Fact 6 with $\ell=t-1$, the number of ways for a walk in $\dot{\mathcal{F}}_{s}^{t}$ to hit $(s, t+s-1)$, and so $(s, t+s)$, is $\left(\begin{array}{c}t+2 s-1 \\ s\end{array}\right)-\left(\begin{array}{c}t+2 s-1 \\ s-1\end{array}\right)$. Further, of such walks, those that hit $(s, t-1)$ are in $\mathcal{W}$, and this can happen in $\left(\begin{array}{c}t+s-1 \\ s\end{array}\right)$ ways. Therefore, looking at only the first $t+2 s$ steps, we see that

$$
\begin{aligned}
\mu_{p}(\dot{\mathcal{A}}) & \leq \mu_{p}\left(\dot{\mathcal{F}}_{s}^{t} \backslash \mathcal{W}\right) \leq\left(\left(\begin{array}{c}
t+2 s-1 \\
s
\end{array}\right)-\left(\begin{array}{c}
t+2 s-1 \\
s-1
\end{array}\right)-\left(\begin{array}{c}
t+s-1 \\
s
\end{array}\right)\right) p^{t+s} q^{s} \\
& =\Theta\left(t^{s-1} p^{t+s} q^{s}\right)=\Theta\left((t p)^{s-1} p^{t+1}\right)=O\left(p^{t+1}\right)
\end{aligned}
$$

which gives 25 .

Then we use $\mu_{p}(\ddot{\mathcal{A}})+\mu_{p}(\tilde{\mathcal{A}}) \leq \alpha^{t+1}=O\left(p^{t+1}\right)$, and hence, we infer $\mu_{p}(\mathcal{A})=$ $\mu_{p}(\dot{\mathcal{A}})+\mu_{p}(\ddot{\mathcal{A}})+\mu_{p}(\tilde{\mathcal{A}})=O\left(p^{t+1}\right)$. Since $\mu_{p}(\mathcal{B}) \leq \alpha^{v}=O\left(p^{t}\right)$, we have that $\mu_{p}(\mathcal{A}) \mu_{p}(\mathcal{B})=O\left(p^{2 t+1}\right)$. On the other hand, 20 gives $\mu_{p}\left(\mathcal{F}_{r}^{t}\right)^{2}=\Theta\left(p^{2 t}\right)$. Hence, $\mu_{p}(\mathcal{A}) \mu_{p}(\mathcal{B}) \ll \mu_{p}\left(\mathcal{F}_{r}^{t}\right)^{2}$.

Now we assume that both $\mathcal{A}$ and $\mathcal{B}$ contain $D_{s}^{t}(1)$. Then we can define parameters $I, J$ as follows. Since $\mathcal{A}$ is shifted there exists $I$ with $1 \leq I \leq i_{\text {max }}$ such that $D_{s}^{t}(i) \in \mathcal{A}$ for $i \leq I$ and $D_{s}^{t}(i) \notin \mathcal{A}$ for $i>I$. Similarly there is $1 \leq J \leq i_{\text {max }}$ such that $D_{s}^{t}(j) \in \mathcal{B}$ for $j \leq J$ and $D_{s}^{t}(j) \notin \mathcal{B}$ for $j>J$. Based on their values, we consider the following two cases, Case I: $I=J=i_{\max }$, and Case II: Either $I \neq i_{\max }$ or $J \neq i_{\max }$.

Case I. In this case we apply the following to get $\mathcal{A}, \mathcal{B} \subseteq \mathcal{F}_{s}^{t}$.

Claim 14. If $I=i_{\max }$, then $\mathcal{B} \subset \mathcal{F}_{s}^{t}$. If $J=i_{\max }$, then $\mathcal{A} \subset \mathcal{F}_{s}^{t}$.

Proof. By symmetry, it is enough to prove only the first statement. As $I=i_{\max }$, that is, $D_{s}^{t}\left(i_{\text {max }}\right) \in \mathcal{A}$, Fact 7 (ii) gives that $\operatorname{dual}_{t}\left(D_{s}^{t}\left(i_{\max }\right)\right)=[n] \backslash[t+s, t+2 s] \notin \mathcal{B}$. So Fact 7 (i) implies that each walk $B \in \mathcal{B}$ satisfies $B \nrightarrow \operatorname{dual}_{t}\left(D_{s}^{t}\left(i_{\max }\right)\right)$. Note that $\operatorname{dual}_{t}\left(D_{s}^{t}\left(i_{\text {max }}\right)\right)=[t+s-1] \cup[t+2 s+1, n]$, which consists of line segments connecting $(0,0),(0, t+s-1),(s+1, t+s-1)$, and $(s+1, n-s-1)$. Thus each walk $B \in \mathcal{B}$ must hit one of $(0, t+s),(1, t+s), \ldots,(s, t+s)$, which means $|B \cap[t+2 s]| \geq t+s$. Hence, $\mathcal{B} \subset \mathcal{F}_{s}^{t}$ holds.

One can easily check that Theorem 4(a)-(c) follow from Claim 14 . Note that the equality in (a) and (c) holds if and only if $\mathcal{A}=\mathcal{B}=\mathcal{F}_{s}^{t}$.

Case II. First we prove

$$
X_{*} \ll X_{\Delta}-X_{*}
$$


that is, (b) of Theorem 4 holds for $t \geq t_{0}$, where $t_{0}$ depends on $r$ and $\epsilon_{1}$. (Note that we do not assume (9) here.) Since $X_{*}=\mu_{p}\left(\mathcal{A} \backslash \mathcal{F}_{s}^{t}\right)+\mu_{p}\left(\mathcal{B} \backslash \mathcal{F}_{s}^{t}\right)$ and $X_{\Delta}-X_{*}=$ $\mu_{p}\left(\mathcal{F}_{s}^{t} \backslash \mathcal{A}\right)+\mu_{p}\left(\mathcal{F}_{s}^{t} \backslash \mathcal{B}\right)$, it suffices to show that

$$
\begin{aligned}
& \mu_{p}\left(\mathcal{B} \backslash \mathcal{F}_{s}^{t}\right) \ll \mu_{p}\left(\mathcal{F}_{s}^{t} \backslash \mathcal{A}\right), \\
& \mu_{p}\left(\mathcal{A} \backslash \mathcal{F}_{s}^{t}\right) \ll \mu_{p}\left(\mathcal{F}_{s}^{t} \backslash \mathcal{B}\right) .
\end{aligned}
$$

By symmetry we only show (28). If $I=i_{\max }$ then $\mathcal{B} \subset \mathcal{F}_{s}^{t}$ by Claim 14 and 28 holds. So we may assume that $I \neq i_{\max }$, and there exists $D_{s}^{t}(I+1) \in \dot{\mathcal{F}}_{s}^{t} \backslash \mathcal{A}$.

Claim 15. We have $\mu_{p}\left(\mathcal{F}_{s}^{t} \backslash \mathcal{A}\right) \geq \Theta\left(p^{t} q^{I}\right)$ and $\mu_{p}\left(\mathcal{B} \backslash \mathcal{F}_{s}^{t}\right) \leq(q / p)^{t+I}$.

Proof. To prove the first inequality, consider walks $W$ such that $W \rightarrow D_{s}^{t}(I+1)$ and $W$ hits $Q:=(s, t+s)$. Since $D_{s}^{t}(I+1) \notin \mathcal{A}$, we have $W \in \mathcal{F}_{s}^{t} \backslash \mathcal{A}$ by Fact 7 (i). Since $D_{s}^{t}(I+1)$ contains line segments connecting $Q_{-}:=(s, t-1), Q$, and $Q_{+}:=(s+I+1, t+s)$ it follows that $W$ must hit $Q_{-}$and $Q_{+}$. (See Figure 4) The number of walks from $(0,0)$ to $Q_{-}$is $\left({ }^{t+s-1}\right)$, then there is the unique walk passing $Q_{-}, Q$, and $Q_{+}$which hits $(s, t+s)$. So the measure of the family of all such walks $W$ is $\left(\begin{array}{c}t+s-1 \\ s\end{array}\right) p^{t+s} q^{s+I+1}$. After hitting $Q_{+}$, to satisfy $W \rightarrow D_{s}^{t}(I+1)$, walks $W$ must not hit the line $y=x+(t-I)$. This happens with probability at least $1-\alpha$ because the measure of walks starting from $Q_{+}$which hit this line is at most $\alpha$ by (i) of Lemma 5 . Thus we obtain

$$
\mu_{p}\left(\mathcal{F}_{s}^{t} \backslash \mathcal{A}\right) \geq\left(\begin{array}{c}
t+s-1 \\
s
\end{array}\right) p^{t+s} q^{s+I+1}(1-\alpha)=\Theta\left(t^{s} p^{t+s} q^{I}\right)=\Theta\left(p^{t} q^{I}\right) .
$$

Next, we prove the second inequality. Since $D_{s}^{t}(I) \in \mathcal{A}$, we have that $\operatorname{dual}_{t}\left(D_{s}^{t}(I)\right) \notin$ $\mathcal{B}$. Referring to Figure 3 , with $i=I$, one sees that the walk dual ${ }_{t}\left(D_{s}^{t}(I)\right)$ contains line segments connecting $(0,0),(0, t+s-1),(s+1, t+s-1)$, and $(s+1, t+s+I)$; and then from $(s+1, s+t+I)$ the walk never hits the line $y=x+(t+I)$. Hence, by Fact 7 . each walk $B \in \mathcal{B}$ must hit one of $(0, t+s),(1, t+s), \ldots,(s, t+s)$, or $y=x+(t+I)$. Note that all walks hitting one of these $s+1$ points are contained in $\mathcal{F}_{s}^{t}$. Thus, each walk $B \in \mathcal{B} \backslash \mathcal{F}_{s}^{t}$ hits $y=x+(t+I)$. So by Lemma 5 (i) we have

$$
\mu_{p}\left(\mathcal{B} \backslash \mathcal{F}_{s}^{t}\right) \leq \alpha^{t+I}=(p / q)^{t+I},
$$

which completes the proof of the claim.

It follows from the claim that $\frac{\mu_{p}\left(\mathcal{F}_{r}^{t} \backslash \mathcal{A}\right)}{\mu_{p}\left(\mathcal{B} \backslash \mathcal{F}_{r}^{t}\right)} \geq \Theta\left(\frac{p^{t} q^{I}}{(p / q)^{t+1}}\right)=\Theta\left(\left(q^{2} / p\right)^{I}\right) \gg 1$, which yields (28), and then (27). Therefore we have

$$
\begin{aligned}
X & =\mu_{p}(\mathcal{A})+\mu_{p}(\mathcal{B})=X_{*}+\mu_{p}\left(\mathcal{A} \cap \mathcal{F}_{s}^{t}\right)+\mu_{p}\left(\mathcal{B} \cap \mathcal{F}_{s}^{t}\right) \\
& <X_{\Delta}-X_{*}+\mu_{p}\left(\mathcal{A} \cap \mathcal{F}_{s}^{t}\right)+\mu_{p}\left(\mathcal{B} \cap \mathcal{F}_{s}^{t}\right) \leq 2 \mu_{p}\left(\mathcal{F}_{s}^{t}\right)=X_{\mathcal{F}},
\end{aligned}
$$

and so we get (a) of Theorem 4 without equality. Then (c) follows from (a) with the AM-GM inequality, that is, $\mu_{p}(\mathcal{A}) \mu_{p}(\mathcal{B}) \leq(X / 2)^{2}<\left(X_{\mathcal{F}} / 2\right)^{2}=\mu_{p}\left(\mathcal{F}_{s}^{t}\right)^{2}$.

\section{Non-Diagonal EXtremal CASES}

We now deal with the remaining non-diagonal extremal cases $\left(s, s^{\prime}\right)=(r+1, r)$ or $(r, r-1)$. In these cases, we have $s-s^{\prime}=1$, and hence, $u=t-1$, and $v=t+1$. The argument here is similar to that of Section 6 , but it is slightly complicated because of the asymmetry of $u$ and $v$. 
As in Section 6, let

$$
D_{s}^{u}(i)=[u-1] \cup[u+s, u+2 s] \cup[u+2 s+i+2, n]_{2} \in \dot{\mathcal{F}}_{s}^{u},
$$

for $1 \leq i \leq n-u-2 s-1=: i_{\max }$, where $i_{\max }$ is defined so that $D_{s}^{u}\left(i_{\max }\right)=$ $[u-1] \cup[u+s, u+2 s]$. It is not hard to check

$$
\operatorname{dual}_{t}\left(D_{s}^{u}(i)\right)=[u+s] \cup[u+2 s+1, u+2 s+i+1] \cup[u+2 s+i+3, n]_{2}
$$

In particular, $\operatorname{dual}_{t}\left(D_{s}^{u}\left(i_{\max }\right)\right)=\left[v+s^{\prime}-1\right] \cup\left[v+2 s^{\prime}+1, n\right]=[n] \backslash\left[v+s^{\prime}, v+2 s^{\prime}\right]$. On the other hand, $\operatorname{dual}_{t}\left(D_{s^{\prime}}^{v}\left(i_{\max }\right)\right) \neq[n] \backslash[u+s, u+2 s]$ because of the asymmetry of $u$ and $v$. To fix this problem we vary from Section 6 a bit, and let

$$
\widetilde{D}_{s^{\prime}}^{v}(j):=[v-2] \cup\left[v+s^{\prime}-1, v+2 s^{\prime}\right] \cup\left[v+2 s^{\prime}+j+2, n\right]_{2} \in \dot{\mathcal{F}}_{s^{\prime}}^{v},
$$

for $1 \leq j \leq n-v-2 s^{\prime}-1=n-u-2 s-1=i_{\max }$. Then,

$$
\begin{aligned}
\operatorname{dual}_{t}\left(\widetilde{D}_{s^{\prime}}^{v}(j)\right) & =\left[v+s^{\prime}-2\right] \cup\left[v+2 s^{\prime}+1, v+2 s^{\prime}+j+1\right] \cup\left[v+2 s^{\prime}+j+3, n\right]_{2} \\
& =[u+s-1] \cup[u+2 s+1, u+2 s+j+1] \cup[u+2 s+j+3, n]_{2},
\end{aligned}
$$

and $\operatorname{dual}_{t}\left(\widetilde{D}_{s^{\prime}}^{v}\left(i_{\max }\right)\right)=[n] \backslash[u+s, u+2 s]$, as desired. We use $D_{s}^{u}(i)$ for $\mathcal{A}$ and $\widetilde{D}_{s^{\prime}}^{v}(j)$ for $\mathcal{B}$.

First we show that if $D_{s}^{u}(1) \notin \mathcal{A}$ or $\widetilde{D}_{s^{\prime}}^{v}(1) \notin \mathcal{B}$, then Theorem 4 vacuously holds since (9) does not hold.

Lemma 16. If $D_{s}^{u}(1) \notin \mathcal{A}$ or $\widetilde{D}_{s^{\prime}}^{v}(1) \notin \mathcal{B}$, then $\mu_{p}(\mathcal{A}) \mu_{p}(\mathcal{B}) \ll \mu_{p}\left(\mathcal{F}_{r}^{t}\right)^{2}$, that is, there exists $t_{0}$ depending on $r$ and $\delta_{1}$ such that $\mu_{p}(\mathcal{A}) \mu_{p}(\mathcal{B})<\left(1-\delta_{1}\right) \mu_{p}\left(\mathcal{F}_{r}^{t}\right)^{2}$ for all $t \geq t_{0}$.

Proof. If $D_{s}^{u}(1) \notin \mathcal{A}$ then replacing $t$ with $u$ in 26) gives

$\mu_{p}(\dot{\mathcal{A}}) \leq\left(\left(\begin{array}{c}u+2 s-1 \\ s\end{array}\right)-\left(\begin{array}{c}u+2 s-1 \\ s-1\end{array}\right)-\left(\begin{array}{c}u+s-1 \\ s\end{array}\right)\right) p^{u+s} q^{s}=O\left(p^{u+1}\right)=O\left(p^{t}\right)$.

This together with $\mu_{p}(\ddot{\mathcal{A}})+\mu_{p}(\tilde{\mathcal{A}}) \leq \alpha^{u+1}=O\left(p^{t}\right)$ yields $\mu_{p}(\mathcal{A})=O\left(p^{t}\right)$. For $\mathcal{B}$ we use the obvious estimation $\mu_{p}(\mathcal{B}) \leq \alpha^{v}=O\left(p^{t+1}\right)$. Thus $\mu_{p}(\mathcal{A}) \mu_{p}(\mathcal{B})=O\left(p^{2 t+1}\right)$.

If $\widetilde{D}_{s^{\prime}}^{v}(1) \notin \mathcal{B}$ then we have $\mu_{p}(\dot{\mathcal{B}})=O\left(p^{t+2}\right)$. Indeed, with only obvious changes to the proof of Lemma 13 , we get that $\dot{\mathcal{B}} \subset \dot{\mathcal{F}}_{s^{\prime}}^{v} \backslash \mathcal{W}^{\prime}$ where $\mathcal{W}^{\prime}:=\left\{W \in \dot{\mathcal{F}}_{s^{\prime}}^{v}: W \rightarrow\right.$ $\widetilde{D}_{s^{\prime}}^{v}(1), W$ hits $\left.\left(s^{\prime}, v+s^{\prime}\right)\right\}$, and, using $\mu_{p}(\dot{\mathcal{B}}) \leq \mu_{p}\left(\dot{\mathcal{F}}_{s^{\prime}}^{v} \backslash \mathcal{W}^{\prime}\right)$, we have that

$$
\begin{aligned}
\mu_{p}(\dot{\mathcal{B}}) & \leq\left(\left(\begin{array}{c}
v+2 s^{\prime}-1 \\
s^{\prime}
\end{array}\right)-\left(\begin{array}{c}
v+2 s^{\prime}-1 \\
s^{\prime}-1
\end{array}\right)-\left(\begin{array}{c}
v+s^{\prime}-1 \\
s^{\prime}
\end{array}\right)\right) p^{v+s^{\prime}} q^{s^{\prime}} \\
& =O\left(p^{v+1}\right)=O\left(p^{t+2}\right) .
\end{aligned}
$$

(If $s^{\prime}=0$ then $\dot{\mathcal{F}}_{s^{\prime}}^{v}=\mathcal{W}^{\prime}$ and $\mu_{p}(\dot{\mathcal{B}})=0$.) Thus $\mu_{p}(\mathcal{B})=O\left(p^{t+2}\right)$ follows from $\mu_{p}(\ddot{\mathcal{B}})+\mu_{p}(\tilde{\mathcal{B}}) \leq \alpha^{v+1}=O\left(p^{t+2}\right)$. Since $\mu_{p}(\mathcal{A}) \leq \alpha^{u}=O\left(p^{t-1}\right)$ we get $\mu_{p}(\mathcal{A}) \mu_{p}(\mathcal{B})=$ $O\left(p^{2 t+1}\right)$.

Consequently if $D_{s}^{u}(1) \notin \mathcal{A}$ or $\tilde{D}_{s^{\prime}}^{v} \notin \mathcal{B}$ then $\mu_{p}(\mathcal{A}) \mu_{p}(\mathcal{B})=O\left(p^{2 t+1}\right) \ll \mu_{p}\left(\mathcal{F}_{r}^{t}\right)^{2}$.

Now we assume that both $\mathcal{A}$ and $\mathcal{B}$ contain $D_{s}^{t}(1)$. We define the parameters $I:=\max \left\{i \geq 1: D_{s}^{u}(i) \in \mathcal{A}\right\}$ and $J:=\max \left\{j \geq 1: \widetilde{D}_{s^{\prime}}^{v}(j) \in \mathcal{B}\right\}$. We again have the following two cases, that is, Case I: $I=J=i_{\max }$, and Case II: Either $I \neq i_{\max }$ or $J \neq i_{\max }$.

Case I. In this case we have $D_{s}^{u}\left(i_{\max }\right) \in \mathcal{A}$ and $\widetilde{D}_{s^{\prime}}^{v}\left(i_{\max }\right) \in \mathcal{B}$. We can argue as in the previous section. The assumption $D_{s}^{u}\left(i_{\max }\right) \in \mathcal{A}$ implies that $\operatorname{dual}_{t}\left(D_{s}^{u}\left(i_{\max }\right)\right)=$ 
$[n] \backslash\left[v+s^{\prime}, v+2 s^{\prime}\right] \notin \mathcal{B}$, and hence, $\mathcal{B} \subset \mathcal{F}_{s^{\prime}}^{v}$. Similarly $\widetilde{D}_{s^{\prime}}^{v}\left(i_{\max }\right) \in \mathcal{B}$ implies $\mathcal{A} \subset \mathcal{F}_{s}^{u}$. One can easily check that Theorem 4 (a)-(c) follow from $\mathcal{B} \subset \mathcal{F}_{s^{\prime}}^{v}$ and $\mathcal{A} \subset \mathcal{F}_{s}^{u}$.

Case II. First we prove

$$
X_{*} \ll X_{\Delta}-X_{*},
$$

that is, (b) of Theorem 4 holds for $t \geq t_{0}$, where $t_{0}$ depends on $r$ and $\epsilon_{1}$. To this end it suffices to show the following.

Claim 17. We have $\frac{1}{p} \mu_{p}\left(\mathcal{B} \backslash \mathcal{F}_{s^{\prime}}^{v}\right) \ll p \mu_{p}\left(\mathcal{F}_{s}^{u} \backslash \mathcal{A}\right)$ and $p \mu_{p}\left(\mathcal{A} \backslash \mathcal{F}_{s}^{u}\right) \ll \frac{1}{p} \mu_{p}\left(\mathcal{F}_{s^{\prime}}^{v} \backslash \mathcal{B}\right)$.

Proof. First, we show the first inequality. If $I=i_{\max }$ then this is true because $\mathcal{B} \subset \mathcal{F}_{s^{\prime}}^{v}$ and $\mu_{p}\left(\mathcal{B} \backslash \mathcal{F}_{s^{\prime}}^{v}\right)=0$ follow from the argument in Case I. So assume that $I \neq i_{\text {max }}$. Changing $t$ to $u$ in the argument used to give (29) yields

$$
\mu_{p}\left(\mathcal{F}_{s}^{u} \backslash \mathcal{A}\right) \geq\left(\begin{array}{c}
u+s-1 \\
s
\end{array}\right) p^{u+s} q^{s+I+1}(1-\alpha) \geq \Theta\left(u^{s} p^{u+s} q^{I}\right)=\Theta\left(p^{t-1} q^{I}\right),
$$

and changing $t$ to $v$ in the argument for 30 gives $\mu_{p}\left(\mathcal{B} \backslash \mathcal{F}_{s^{\prime}}^{v}\right) \leq \alpha^{v+I}=(p / q)^{t+I+1}$. Thus

$$
\frac{p \mu_{p}\left(\mathcal{F}_{s}^{u} \backslash \mathcal{A}\right)}{\frac{1}{p} \mu_{p}\left(\mathcal{B} \backslash \mathcal{F}_{s^{\prime}}^{v}\right)} \geq \Theta\left(\frac{p^{t-1} q^{I}}{(p / q)^{t+I+1}}\right)=\Theta\left(\left(q^{2} / p\right)^{I} p^{-2}\right) \gg 1,
$$

showing the first inequality.

Next, we prove the second inequality. This is clearly true if $J=i_{\max }$. So assume that $J \neq i_{\max }$. We have

$$
\widetilde{D}_{s^{\prime}}^{v}(J+1)=[v-2] \cup\left[v+s^{\prime}-1, v+2 s^{\prime}\right] \cup\left[v+2 s^{\prime}+J+2, n\right]_{2} \notin \mathcal{B},
$$

and walks which hit $\left(s^{\prime}, v+s^{\prime}\right)$ and shift to $\widetilde{D}_{s^{\prime}}^{v}(J+1)$ belong to $\mathcal{F}_{s^{\prime}}^{v} \backslash \mathcal{B}$. These walks must hit $\left(s^{\prime}, v-2\right)$, then $\left(s^{\prime}, v+s^{\prime}\right)$ and then $Q_{+}=\left(s^{\prime}+J+1, v+s^{\prime}\right)$, and after hitting $Q_{+}$they never hit the line $y=x+(v-J)$. Thus

$$
\mu_{p}\left(\mathcal{F}_{s^{\prime}}^{v} \backslash \mathcal{B}\right) \geq\left(\begin{array}{c}
v+s^{\prime}-2 \\
s^{\prime}
\end{array}\right) p^{v+s^{\prime}} q^{s^{\prime}+J+1}(1-\alpha) \geq \Theta\left(v^{s^{\prime}} p^{v+s^{\prime}} q^{J}\right)=\Theta\left(p^{t+1} q^{J}\right),
$$

while as $\operatorname{dual}_{t}\left(\widetilde{D}_{s^{\prime}}^{v}(J)\right)=[u+s-1] \cup[u+2 s+1,, u+2 s+J+1] \cup[u+2 s+J+3, n]_{2} \notin \mathcal{A}$ implies, following the argument for $(30)$, that each walk in $\mathcal{A} \backslash \mathcal{F}_{s}^{u}$ hits $y=x+(u+J)$. Together this gives $\mu_{p}\left(\mathcal{A} \backslash \mathcal{F}_{s}^{u}\right) \leq \alpha^{u+J}=(p / q)^{t-1+J}$. So

$$
\frac{\frac{1}{p} \mu_{p}\left(\mathcal{F}_{s^{\prime}}^{v} \backslash \mathcal{B}\right)}{p \mu_{p}\left(\mathcal{A} \backslash \mathcal{F}_{s}^{u}\right)} \geq \Theta\left(\frac{p^{t+1} q^{J}}{(p / q)^{t-1+J}}\right)=\Theta\left(\left(q^{2} / p\right)^{J} p^{-2}\right) \gg 1
$$

which proves the second inequality.

Next we check that (a) of Theorem 4 holds without equality. Indeed it follows from (31) that

$$
\begin{aligned}
X & =p \mu_{p}(\mathcal{A})+\frac{1}{p} \mu_{p}(\mathcal{B})=X_{*}+p \mu_{p}\left(\mathcal{A} \cap \mathcal{F}_{s}^{t}\right)+\frac{1}{p} \mu_{p}\left(\mathcal{B} \cap \mathcal{F}_{s}^{t}\right) \\
& <X_{\Delta}-X_{*}+p \mu_{p}\left(\mathcal{A} \cap \mathcal{F}_{s}^{t}\right)+\frac{1}{p} \mu_{p}\left(\mathcal{B} \cap \mathcal{F}_{s}^{t}\right) \leq X_{\mathcal{F}}
\end{aligned}
$$

Finally we verify (c) of Theorem 4 .

Lemma 18. We have $\mu_{p}(\mathcal{A}) \mu_{p}(\mathcal{B})<\mu_{p}\left(\mathcal{F}_{s}^{u}\right) \mu_{p}\left(\mathcal{F}_{s^{\prime}}^{v}\right)$ for all $t \geq t_{0}$, where $t_{0}$ depends on $r, \epsilon_{1}, \epsilon$. 
Proof. Let

$$
\begin{array}{lll}
f=p \mu_{p}\left(\mathcal{F}_{s}^{u}\right), & f_{*}=p \mu_{p}\left(\mathcal{F}_{s}^{u} \backslash \mathcal{A}\right), & a_{*}=p \mu_{p}\left(\mathcal{A} \backslash \mathcal{F}_{s}^{u}\right), \\
g=\mu_{p}\left(\mathcal{F}_{s^{\prime}}^{v}\right) / p, & g_{*}=\mu_{p}\left(\mathcal{F}_{s^{\prime}}^{v} \backslash \mathcal{B}\right) / p, & b_{*}=\mu_{p}\left(\mathcal{B} \backslash \mathcal{F}_{s^{\prime}}^{v}\right) / p .
\end{array}
$$

Let $\beta=\epsilon / 2$ for $r=0$, and let $\beta$ be any fixed constant satisfying $0<\beta<\frac{r}{r+1}$ for $r \geq 1$. Claim 17 gives that $b_{*}<\beta f_{*}$ and $a_{*}<\beta g_{*}$. So

$$
p \mu_{p}(\mathcal{A})=f-f_{*}+a_{*}<f-f_{*}+\beta g_{*}, \quad \mu_{p}(\mathcal{B}) / p=g-g_{*}+b_{*}<g-g_{*}+\beta f_{*},
$$

and hence, $\mu_{p}(\mathcal{A}) \mu_{p}(\mathcal{B})<\left(f-f_{*}+\beta g_{*}\right)\left(g-g_{*}+\beta f_{*}\right)$. Therefore, in order to show Lemma 18, it suffices to show that

$$
\left(f-\left(f_{*}-\beta g_{*}\right)\right)\left(g-\left(g_{*}-\beta f_{*}\right)\right) \leq f g .
$$

If both $f_{*}-\beta g_{*}$ and $g_{*}-\beta f_{*}$ are non-negative, then we clearly have 32 . If both of them are negative or 0 , then $f_{*} \leq \beta g_{*} \leq \beta^{2} f_{*}$. This implies $f_{*}=g_{*}=0$, and hence, we also get (32). Thus we may assume that $\left(f_{*}-\beta g_{*}\right)\left(g_{*}-\beta f_{*}\right)<0$, and by symmetry, $f_{*}-\beta g_{*}<0<g_{*}-\beta f_{*}$. Since $0<\beta<1$ we have $0<\beta f_{*}<\beta^{-1} f_{*}$ and $g_{*}-\beta f_{*}>g_{*}-\beta^{-1} f_{*}$. Also we have $0<\beta g_{*}-f_{*}=\beta\left(g_{*}-\beta^{-1} f_{*}\right)<g_{*}-\beta^{-1} f_{*}$. Consequently we have $g_{*}-\beta f_{*}>g_{*}-\beta^{-1} f_{*}>0$ and

$$
\frac{\beta g_{*}-f_{*}}{g_{*}-\beta f_{*}}<\frac{\beta g_{*}-f_{*}}{g_{*}-\beta^{-1} f_{*}}=\beta \text {. }
$$

Since (32) is rewritten as $\left(f_{*}-\beta g_{*}\right)\left(g_{*}-\beta f_{*}\right) \leq f\left(g_{*}-\beta f_{*}\right)-g\left(\beta g_{*}-f_{*}\right)$ and we know that the LHS is negative, in order to prove (32) it suffices to show that the RHS is non-negative, that is,

$$
f\left(g_{*}-\beta f_{*}\right) \geq g\left(\beta g_{*}-f_{*}\right) .
$$

On the other hand, 19 implies that

$$
\frac{f}{g}=\frac{\left(\begin{array}{c}
u+2 s \\
s
\end{array}\right) p^{u+s} q^{s}}{\left(\begin{array}{c}
v+2 s^{\prime} \\
s^{\prime}
\end{array}\right) p^{v+s^{\prime}} q^{s^{\prime}}} p^{2}(1+o(1))=\frac{(u+2 s) !\left(v+s^{\prime}\right) ! s^{\prime} !}{\left(v+2 s^{\prime}\right) !(u+s) ! s !} p q(1+o(1))=\frac{t+s}{s} p q(1+o(1)) .
$$

If $r \geq 1$, then using $u=t-1, v=t+1, s^{\prime}=s-1$, and noting that $p q$ attains its minimum at $p=\frac{r}{t+2 r-1}$, we get

$$
\frac{f}{g}=\frac{t+s}{s} p q(1+o(1)) \geq \frac{t+r+1}{r+1} \frac{r}{t+2 r-1} \frac{t+r-1}{t+2 r-1}(1+o(1))=\frac{r}{r+1}(1+o(1))>\beta .
$$

Similarly, if $r=0$, then $\left(s, s^{\prime}\right)=(1,0)$, that is, $s=1$, and

$$
\frac{f}{g}=(t+1) p q(1+o(1)) \geq(t+1) \frac{\epsilon}{t}\left(1-\frac{\epsilon}{t}\right)(1+o(1))=\epsilon(1+o(1))>\beta .
$$

In both cases we have $\frac{f}{g}>\beta$, and this together with 33 yields (34) as needed.

\section{CONCLUding REMARKS}

Recently, Ellis, Keller and Lifshitz [7] obtained a sharp stability result for $t$ intersecting families. The following is Theorem 1.10 of 7 with only minimal changes of notation.

Theorem 5 ([7]). For any $t \in \mathbb{N}$ and any $\xi>0$, there exists $C=C(t, \xi)>0$ such that the following holds. Let $\frac{r}{t+2 r-1}+\xi<p<\frac{r+1}{t+2 r+1}-\xi$, and let $\epsilon>0$. If $\mathcal{F} \subset 2^{[n]}$ is a t-intersecting family such that $\mu_{p}(\mathcal{F}) \geq(1-\epsilon) \mu_{p}\left(\mathcal{F}_{r}^{t}\right)$, then there exists a family $\mathcal{G}$ isomorphic to $\mathcal{F}_{r}^{t}$ such that $\mu_{p}(\mathcal{F} \backslash \mathcal{G}) \leq C \epsilon^{\log _{1-p} p}$. 
Their result is related to Theorem 3 for the case $\mathcal{A}=\mathcal{B}$. To make a comparison easier we state a version of our Theorem 3 , which can be proved almost exactly as Theorem 3 is proved.

Theorem 6. For every integer $r \geq 0$ and all real numbers $\epsilon \in(0,1 / 2)$ and $C>2$, there exists an integer $t_{0}=t_{0}(r, \epsilon, C)$ such that for all $n \geq t \geq t_{0}$ the following holds. Let $\frac{r+\epsilon}{t} \leq p \leq \frac{r+1-\epsilon}{t}$. If $\mathcal{A}, \mathcal{B} \subset 2^{[n]}$ are $t$-nice families such that $\sqrt{\mu_{p}(\mathcal{A}) \mu_{p}(\mathcal{B})} \geq$ $(1-\gamma) \mu_{p}\left(\mathcal{F}_{r}^{t}\right)$ with $\gamma \in\left(0, \frac{\epsilon}{2(r+1)}\right)$, then

$$
\mu_{p}\left(\mathcal{A} \triangle \mathcal{F}_{r}^{t}\right)+\mu_{p}\left(\mathcal{B} \triangle \mathcal{F}_{r}^{t}\right) \leq C \mu_{p}\left(\mathcal{F}_{r}^{t}\right)
$$

In particular, if $\mathcal{A}=\mathcal{B}$ then $\mu_{p}(\mathcal{A}) \geq(1-\gamma) \mu_{p}\left(\mathcal{F}_{r}^{t}\right)$ implies $\mu_{p}\left(\mathcal{A} \triangle \mathcal{F}_{r}^{t}\right) \leq(C / 2) \mu_{p}\left(\mathcal{F}_{r}^{t}\right)$.

Their setup is different from ours and it seems that neither result implies the other. We should note that their results apply to all (not necessarily shifted) $t$-intersecting families. It would be very interesting to see whether one can use their technique to remove the shiftedness condition from Theorem 4, See also 7, 14, for related stability results for intersecting families.

\section{REFERENCES}

[1] R. Ahlswede, L.H. Khachatrian. The diametric theorem in Hamming spaces-optimal anticodes. Adv. in Appl. Math. 20 (1998) 429-449. https://doi.org/10.1006/aama.1998.0588

[2] C. Bey, K. Engel. Old and new results for the weighted t-intersection problem via AK-methods. Numbers, Information and Complexity, Althofer, Ingo, Eds. et al., Dordrech, Kluwer Academic Publishers (2000) 45-74. https://doi.org/10.1007/978-1-4757-6048-4_5

[3] P. Borg. The maximum product of sizes of cross-t-intersecting uniform families. Australas. J. Combin., 60/1 (2014) 69-78. link

[4] P. Borg. The maximum product of weights of cross-intersecting families. J. Lond. Math. Soc. 94/2 (2016) 993-1018. https://doi.org/10.1112/jlms/jdw067

[5] P. Borg, The maximum product of sizes of cross-intersecting families. Discrete Math. 340/9 (2017) 2307-2317. https://arxiv.org/abs/1605.08752

[6] I. Dinur, S. Safra. On the Hardness of Approximating Minimum Vertex-Cover. Ann. of Math. 162 (2005) 439-485. https://doi.org/10.4007/annals.2005.162.439

[7] D. Ellis, N. Keller, N. Lifshitz. Stability versions of Erdös-Ko-Rado type theorems, via isoperimetry. https://arxiv.org/abs/1604.02160

[8] D. Ellis, N. Keller, N. Lifshitz. Stability for the Complete Intersection Theorem, and the Forbidden Intersection Problem of Erdös and Sós. https://arxiv.org/abs/1604.06135v4

[9] Y. Filmus. The weighted complete intersection theorem. J. Combin. Theory (A) 151 (2017) 84-101. https://doi.org/10.1016/j.jcta.2017.04.008

[10] P. Frankl. The Erdös-Ko-Rado theorem is true for $n=c k t$. Combinatorics (Proc. Fifth Hungarian Colloq., Keszthey, 1 (1976), 365-375) Colloq. math. Soc. János Bolyai, 18, North-Holland, 1978.

[11] P. Frankl. The shifting technique in extremal set theory. Surveys in combinatorics (New Cross, 1987) 81-110, London Math. Soc. Lecture Note Ser. 123. http://www.renyi.hu/ pfrankl/ $1987-4 . \mathrm{pdf}$

[12] P. Frankl, Z. Füredi. Beyond the Erdös-Ko-Rado theorem. J. Combin. Theory (A) 56 (1991) 182-194. https://doi.org/10.1016/0097-3165(91)90031-B

[13] P. Frankl, S. J. Lee, M. Siggers, N. Tokushige, An Erdős-Ko-Rado theorem for cross $t$ intersecting families. J. Combin. Theory (A) 128 (2014) 207-249. https://doi.org/10.1016/ j.jcta.2014.08.006

[14] E. Friedgut. On the measure of intersecting families, uniqueness and stability. Combinatorica 28 (2008) 503-528. https://link.springer.com/article/10.1007/s00493-008-2318-9

[15] S. J. Lee, M. Siggers, N. Tokushige, Towards extending the Ahlswede-Khachatrian theorem to cross t-intersecting families. Discrete Appl. Math. 216 (2017) 627-645. https://doi.org/10. $1016 / \mathrm{j}$. dam.2016.02.015

[16] N. Tokushige. Intersecting families - uniform versus weighted. Ryukyu Math. J. 18 (2005) 89103. https://pdfs.semanticscholar.org/39ca/524f1b8fd38f02d27f0853db556014ff10f2.pdf 
Department of Mathematics, Kyung Hee University, Seoul 02447, South Korea

E-mail address: sjlee242@khu.ac.kr

College of Natural Sciences, Kyungpook National University, Daegu 702-701, South KOREA

E-mail address: mhsiggers@knu.ac.kr

College of Education, Ryukyu University, Nishinara, Okinawa 903-0213, Japan

E-mail address: hide@edu.u-ryukyu.ac.jp 